\title{
Pyclen based Ln(III) complexes as highly \\ Page I luminescent bioprobes for in vitro and in vivo one- and two-photon bioimaging applications
}

Nadège Hamon, ${ }^{\dagger}$ Amandine Roux, ${ }^{\ddagger}$ Maryline Beyler, ${ }^{\dagger}$ Jean-Christophe Mulatier, ${ }^{\ddagger}$ Chantal Andraud, ${ }^{\ddagger}$ Christophe Nguyen $\square$, Marie Maynadier, ${ }^{\varsigma}$ Nadir Bettache, $\square$ Alain Duperray, ${ }^{\square}$ Alexei Grichine, ${ }^{\square}$ Sophie Brasselet, ${ }^{\neq}$Magali Gary-Bobo, ${ }^{*} \square$ Olivier Maury* ${ }^{* \ddagger}$ and Raphaël Tripier*†

+ Univ Brest, UMR-CNRS 6521 CEMCA, UFR des Sciences et Techniques, 6 avenue Victor le Gorgeu, C.S. 93837, 29238, Brest, Cedex 3, France. E-mail: raphael.tripier@univ-brest.fr

¥Univ Lyon, ENS de Lyon, CNRS UMR 5182, Université Claude Bernard Lyon 1, Laboratoire de Chimie de l'ENS de Lyon, F-69342 Lyon, France. E-mail: olivier.maury@ens-lyon.fr

口INSERM, U1209, Université Grenoble Alpes, IAB, F-38000, Grenoble, France

* Univ Aix Marseille, CNRS, Centrale Marseille, Institut Fresnel, F-13013 Marseille, France.

- IBMM, Univ Montpellier, CNRS, ENSCM, Montpellier, France. E-Mail: magali.gary-bobo@umontpellier.fr

' NanoMedSyn, 15 Avenue Charles Flahault, 34093, Montpellier Cedex 05, France

- Compound 3

- Figure $\mathrm{S} 1:{ }^{1} \mathrm{H}$ NMR of compound 3

- Figure $\mathrm{S} 2:{ }^{13} \mathrm{C}$ NMR of compound 3

pS3

- Figure S3 : HRMS of compound 3

pS4

- Ligand La

pS5

- Figure $S 4:{ }^{1} \mathrm{H}$ NMR of compound $L^{4 a}$

pS5

- Figure $S 5:{ }^{13} \mathrm{C}$ NMR of compound $L^{4 a}$

pS5

- Figure $S 6:$ HRMS of compound $L^{4 a}$

pS6

- Complex [EuL $\left.{ }^{4 a}\right]$

pS7

- Figure $S 7$ : analytical HPLC of [EuL ${ }^{4 a}$ ]

pS7

- Figure S8 : HRMS of [EuL ${ }^{4 a}$ ]

pS8

- Complex [SmL $\left.{ }^{4 a}\right]$

pS9

- Figure $\mathrm{S9}$ : analytical HPLC of [SmL $\left.{ }^{4 a}\right]$

pS9

- Figure S10 : HRMS of [SmL $\left.{ }^{4 a}\right]$

pS10

- Compound 5

- $\quad$ Figure $\mathrm{S} 11:{ }^{1} \mathrm{H}$ NMR of compound 5

- Figure $\mathrm{S} 12:{ }^{13} \mathrm{C}$ NMR of compound 5

- Figure S13 : HRMS of compound 5

- Figure S14 : analytical HPLC of $L^{4 b}$

- Figure $S 15:{ }^{1} \mathrm{H}$ NMR of compound $L^{4 b}$

- Figure $\mathrm{S} 16:{ }^{13} \mathrm{C}$ NMR of compound $\mathrm{L}^{4 b}$ 
- Figure S17 : HRMS of compound $\mathrm{L}^{4 \mathbf{b}} \quad \mathrm{pS} 15$

- Complex $\left[\mathrm{YbL}^{4 \mathrm{~b}}\right]$

- Figure S18 : analytical HPLC of $\left[\mathrm{YbL}^{4 b}\right]$

pS16

- Figure S19 : HRMS of [YbL $\left.{ }^{4 b}\right]$

pS16

- $\quad$ Compound 7

pS16

pS17

- Figure S20: ${ }^{1} \mathrm{H}$ NMR of compound 7 $\quad$ pS17

- Figure S21: ${ }^{13} \mathrm{C}$ NMR of compound $7 \quad$ pS17

- Figure S22 : HRMS of compound 7 pS18

- Ligand $\mathrm{L}^{4 \mathrm{c}}$

- Figure $\mathrm{S} 23$ : analytical HPLC of $\mathrm{L}^{4 \mathrm{c}}$

- Figure $\mathrm{S} 24:{ }^{1} \mathrm{H}$ NMR of compound $\mathrm{L}^{4 \mathrm{c}}$

pS19

pS19

- Figure $\mathrm{S} 25:{ }^{13} \mathrm{C}$ NMR of compound $\mathrm{L}^{4 c}$

pS19

- Figure S26 : HRMS of compound $\mathrm{L}^{4 c}$

pS20

pS21

- Complex [TbL $\left.{ }^{4 c}\right]$

pS22

- Figure $S 27$ : analytical HPLC of $\left[\mathrm{TbL}^{4 c}\right] \quad$ pS22

- Figure $\mathrm{S} 28$ : HRMS of $\left[\mathrm{TbL}^{4 \mathrm{c}}\right] \quad \mathrm{pS} 23$

- Complex [DyL $\left.{ }^{4 c}\right] \quad$ pS24

- Figure $S 29$ : analytical HPLC of $\left[D^{2} L^{4 c}\right] \quad$ pS24

- Figure S30 : HRMS of [DyL $\left.{ }^{4 c}\right] \quad$ pS25

- Compound $9 \quad$ pS26

- Figure S31: ${ }^{1} \mathrm{H}$ NMR of compound $9 \quad$ pS26

- Figure S32: ${ }^{13} \mathrm{C}$ NMR of compound $9 \quad \mathrm{pS} 26$

- Figure S33 : HRMS of compound $9 \quad$ pS27

- Ligand La' $\quad$ pS28

- Figure S34: ${ }^{1} \mathrm{H}$ NMR of compound $\mathrm{L}^{4 \mathrm{a}^{\prime}} \quad \mathrm{pS} 28$

- Figure S35: ${ }^{13} \mathrm{C}$ NMR of compound $\mathrm{L}^{4 a^{\prime}} \quad \mathrm{pS} 28$

- Figure S36: HRMS of compound $\mathrm{L}^{4 \mathrm{a}^{\prime}} \quad$ pS29

- Complex [EuL $\left.{ }^{4 a^{\prime}}\right] \quad$ pS30

- Figure S37 : analytical HPLC of [EuL4a'] pS30

- Figure $S 38$ : HRMS of [EuL $\left.{ }^{4 a^{\prime}}\right] \quad$ pS31

$\begin{array}{ll}\text { Photophysical measurements } & \text { pS32 }\end{array}$

- Figure S39 : Normalized absorption of of [EuL $\left.{ }^{4 a}\right],\left[\mathrm{SmL}^{4 a}\right],\left[\mathrm{YbL}^{4 b}\right],\left[\mathrm{TbL}^{4 c}\right]$ and $\left[\mathrm{DyL} \mathrm{L}^{4 c}\right]$ in $\mathrm{MeOH} \quad$ pS32

- Figure $\mathrm{S} 40$ : Normalized emission of of [EuL $\left.L^{4 a}\right],\left[\mathrm{SmL}^{4 a}\right],\left[\mathrm{YbL}^{4 b}\right],\left[\mathrm{TbL}^{4 c}\right]$ and $\left[\mathrm{DyL}{ }^{4 c}\right]$ in $\mathrm{MeOH}$ pS33

- Figure S41: Normalized absorption and emission of [EuL $\left.{ }^{4 a}\right]$ PS33

- Figures S42-S44: Fitting of quantum yield experiments PS34

- Figures S45-S53: Exponential lifetime decay measurements PS35 
Page | 3

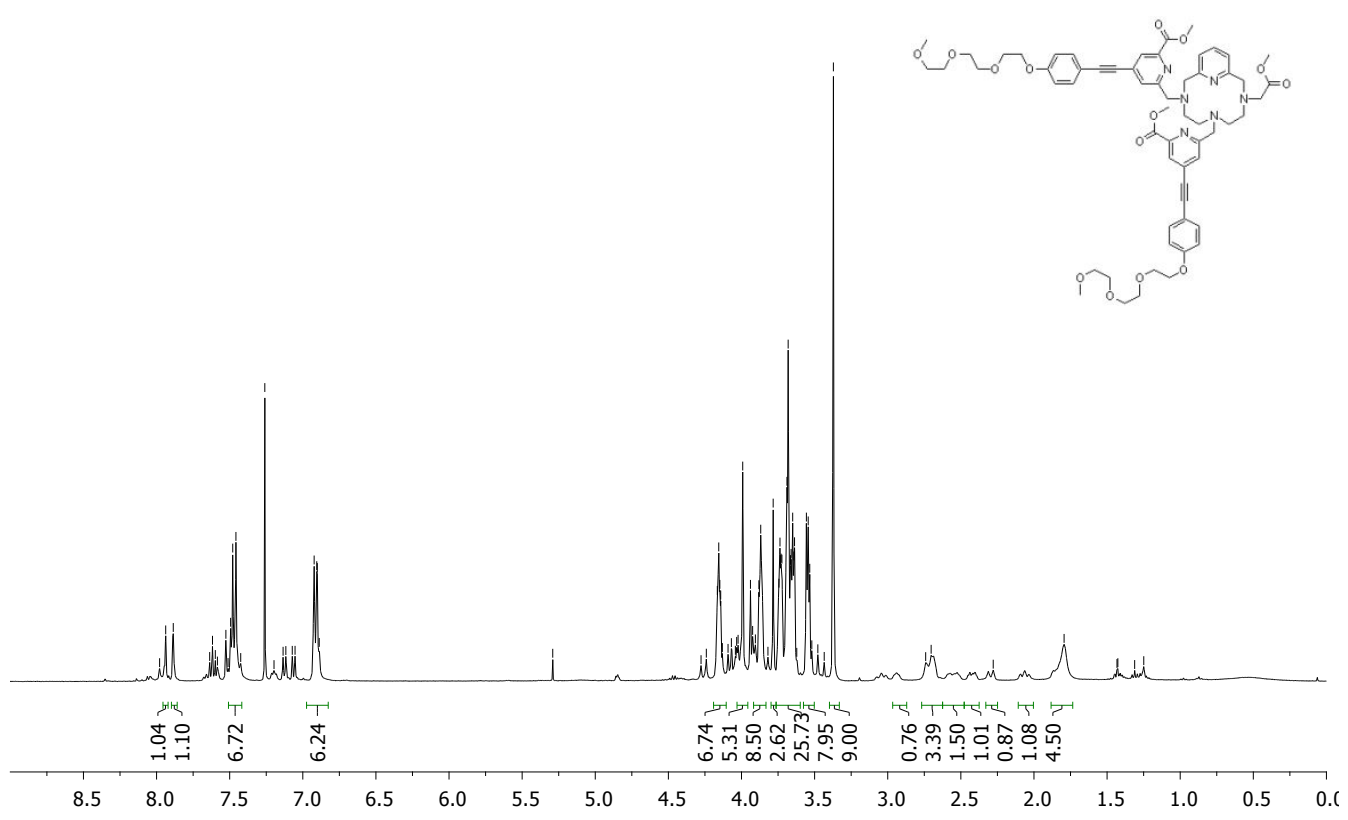

Figure $\mathbf{S 1}:{ }^{1} \mathrm{H}$ NMR of compound 3.

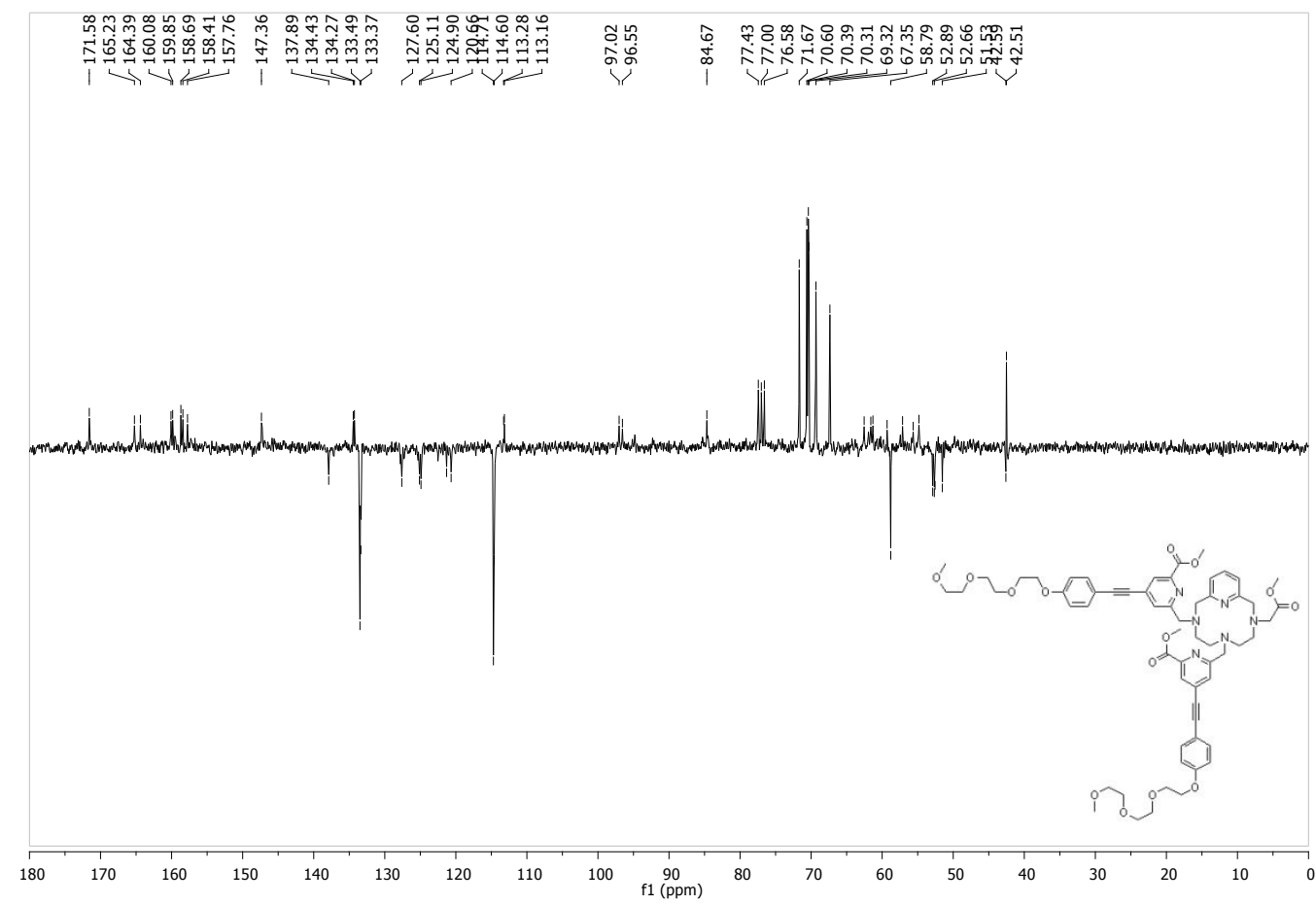

Figure S2 : ${ }^{13} \mathrm{C}$ NMR of compound 3. 
Analysis Info

Sample Name

NH27-f5
X035627CYC.d

Acquisition Date

Instrument / Ser\#

10/07/2017 14:40:18

Analysis Name

Method

maXis 255552.00086

Acquisition Parameter

Source Type
Scan Begin

$\mathrm{ESI}$
$50 \mathrm{~m} / \mathrm{z}$

$2500 \mathrm{~m} / \mathrm{z}$

$\begin{array}{ll}\text { Ion Polarity } & \text { Positive } \\ \text { Set Capillary } & 4500 \mathrm{~V}\end{array}$

Positif.m

Scan End

Set Collision Cell RF

$1800.0 \mathrm{VPp}$

Set Dry Gas

Page | 4
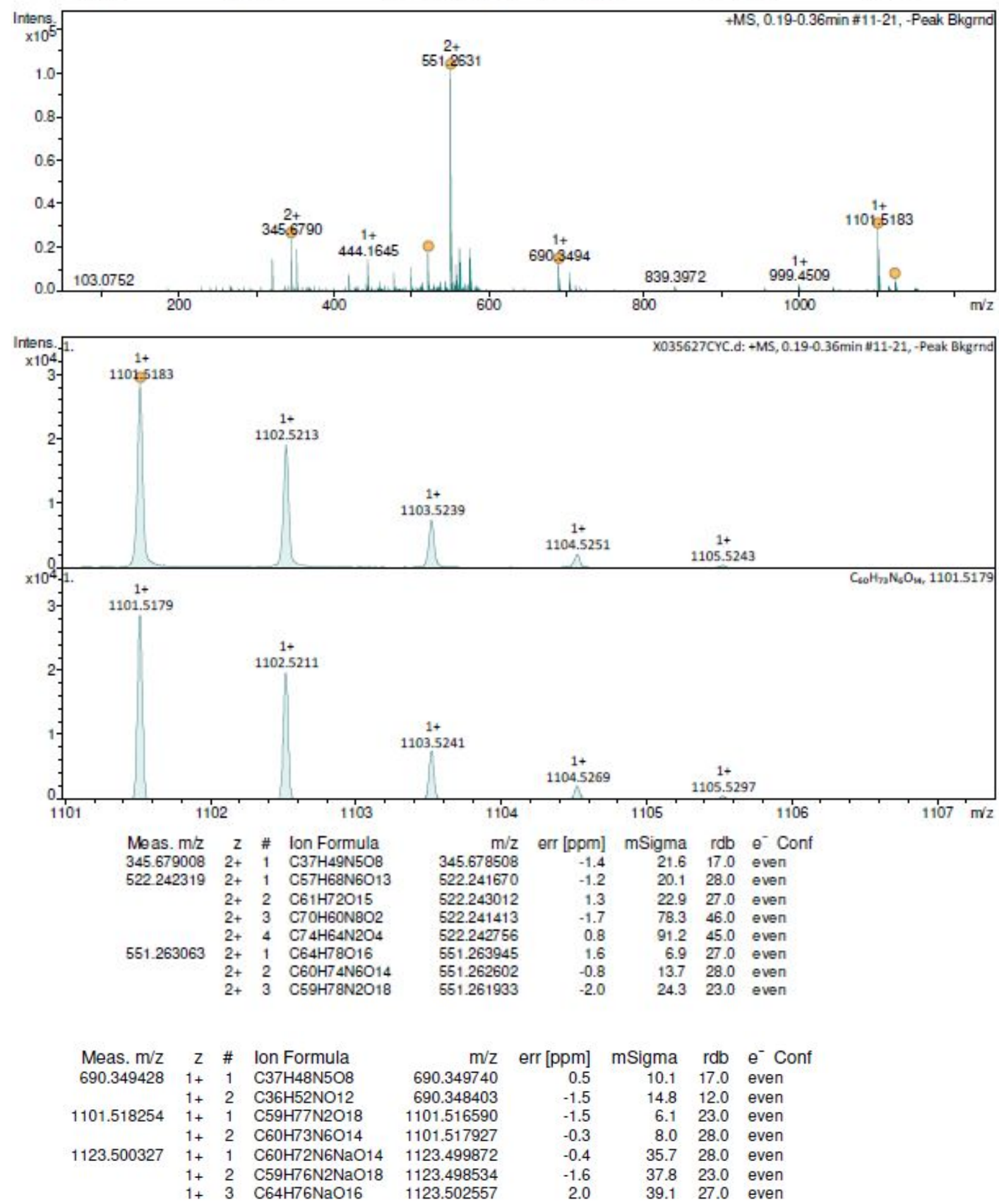

Figure S3 : HRMS of compound 3. 


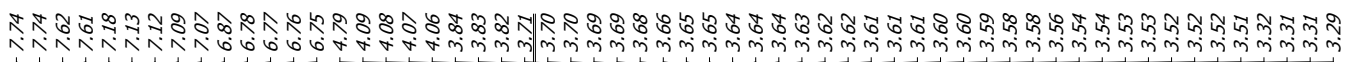

Page | 5

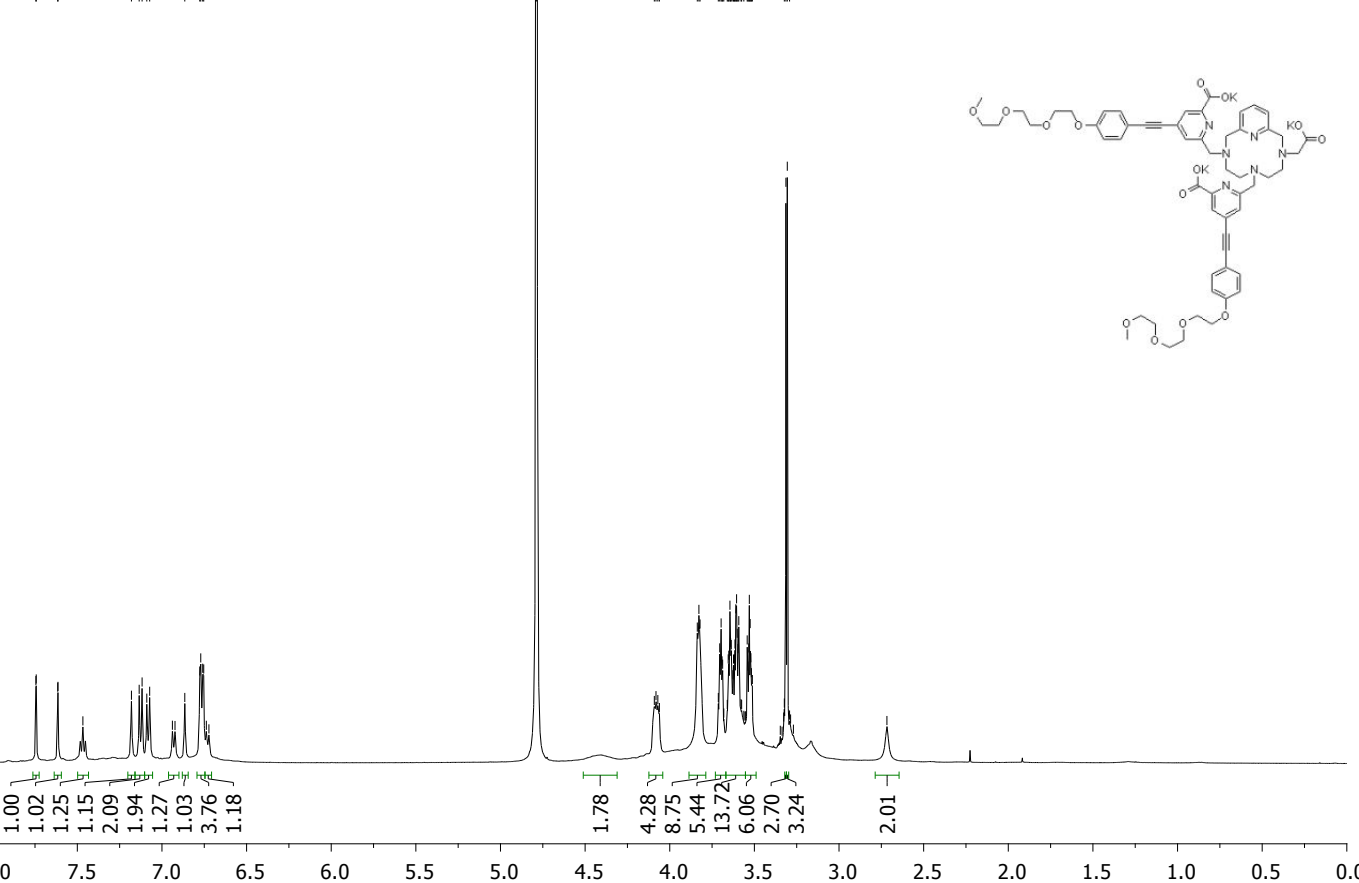

Figure S4 : ${ }^{1} \mathrm{H}$ NMR of compound $\mathrm{L}^{4 \mathrm{a}}$.

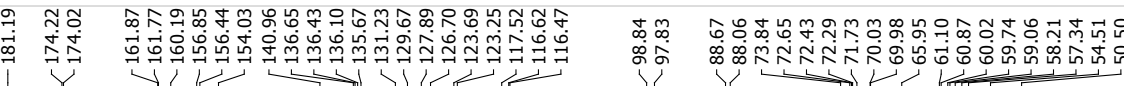

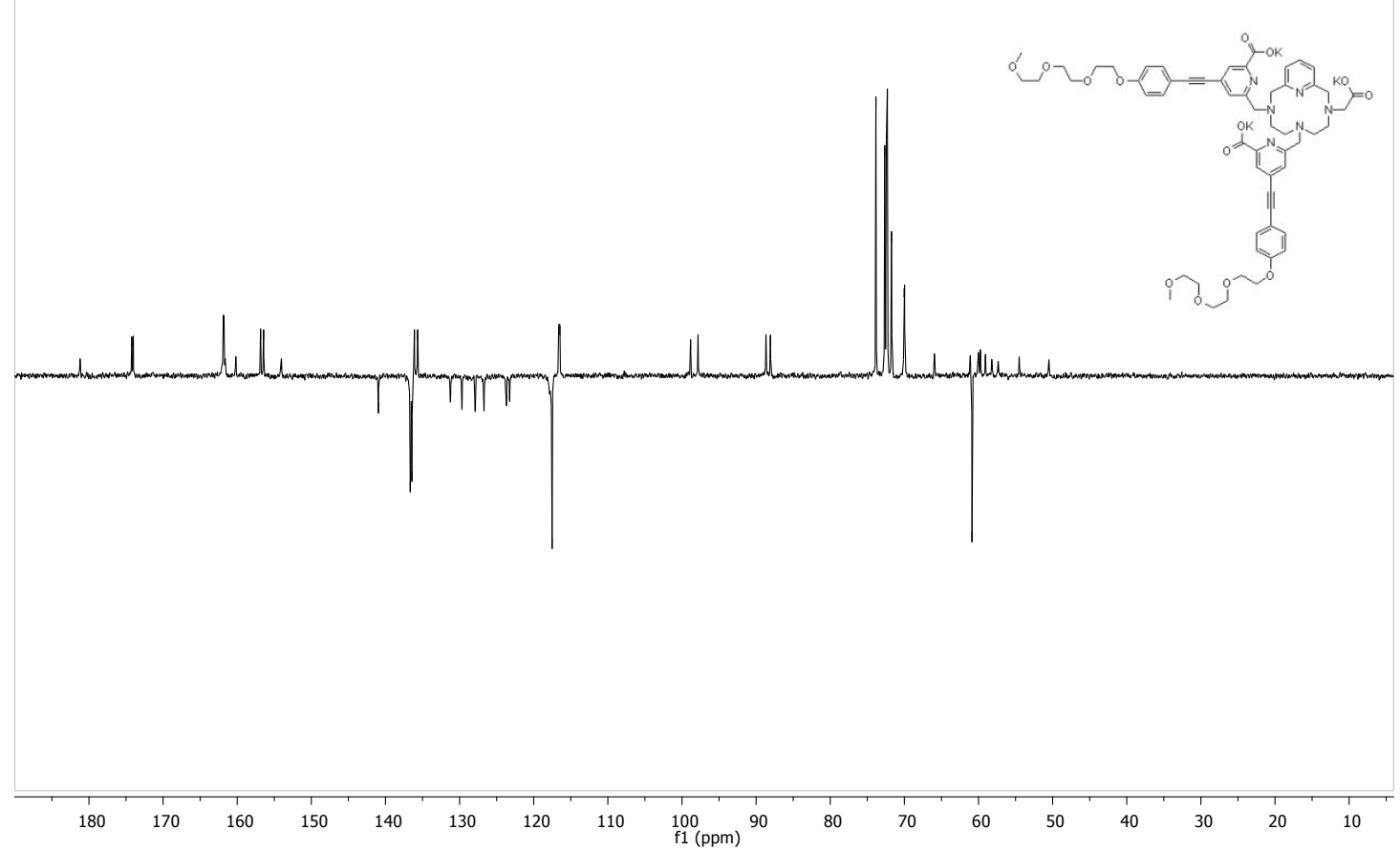

Figure S5 : ${ }^{13} \mathrm{C}$ NMR of compound $\mathrm{L}^{4 \mathrm{a}}$. 

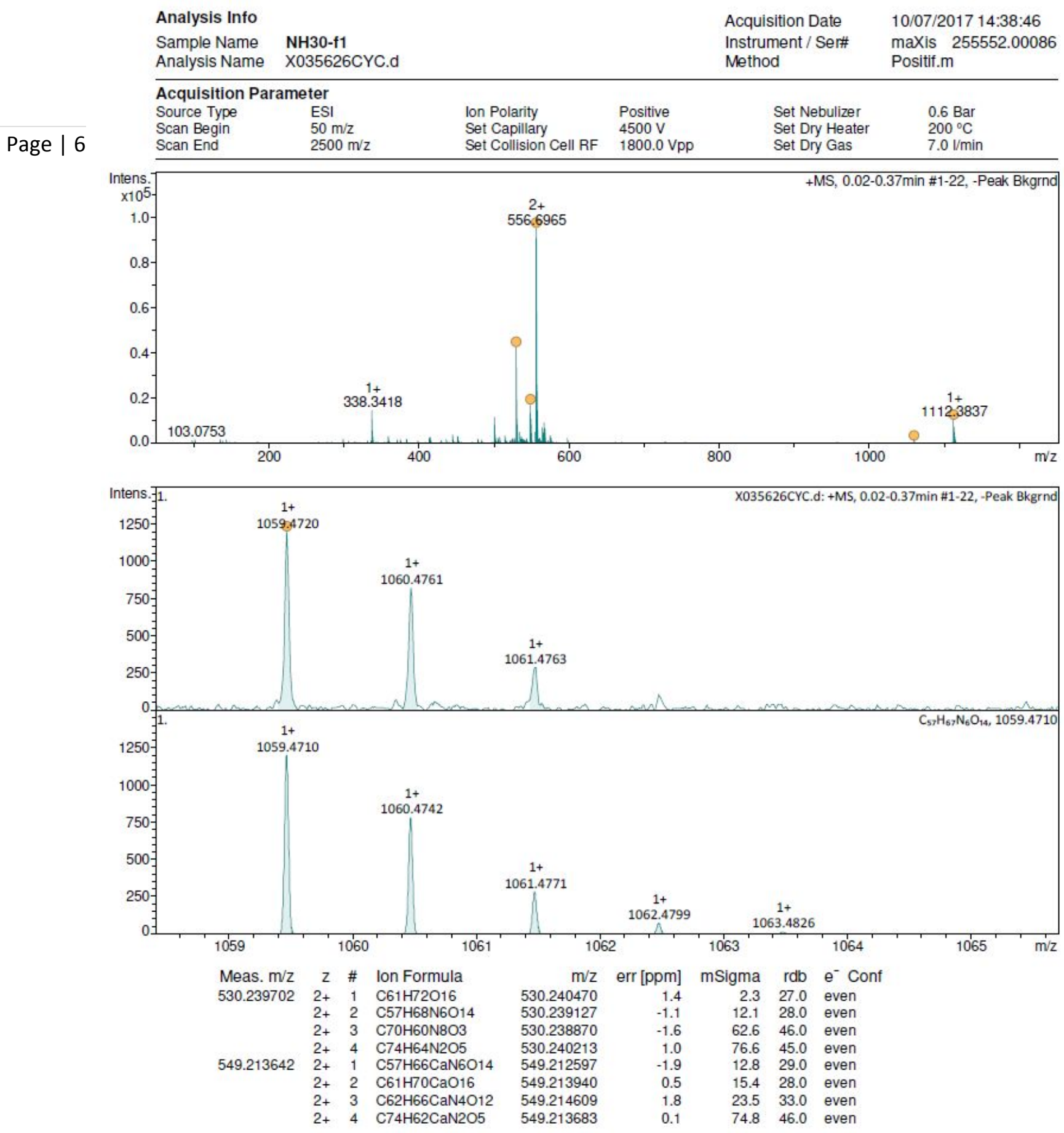


\begin{tabular}{|c|c|c|c|c|c|c|c|c|}
\hline \multirow[t]{2}{*}{ Meas. $\mathrm{m} / \mathrm{z}$} & z & \# & Ion Formula & $\mathrm{m} / \mathrm{z}$ & err [ppm] & mSigma & $\mathrm{rdb}$ & $\mathrm{e}^{-}$Conf \\
\hline & $2+$ & 5 & C75H58CaN6O & 549.214352 & 1.3 & 84.5 & 51.0 & even \\
\hline \multirow[t]{7}{*}{556.696527} & $2+$ & 1 & $\mathrm{C} 61 \mathrm{H} 69 \mathrm{Fe} 016$ & 556.696201 & -0.4 & 8.9 & 28.5 & odd \\
\hline & $2+$ & 2 & C57H65FeN6O14 & 556.694858 & -2.9 & 11.6 & 29.5 & odd \\
\hline & $2+$ & 3 & $\mathrm{C} 62 \mathrm{H} 65 \mathrm{FeN} 4 \mathrm{O} 12$ & 556.696869 & 0.8 & 15.5 & 33.5 & odd \\
\hline & $2+$ & 4 & $\mathrm{C} 63 \mathrm{H} 61 \mathrm{FeN} 8 \mathrm{O} 8$ & 556.697538 & 2.0 & 23.9 & 38.5 & odd \\
\hline & $2+$ & 5 & $\mathrm{C} 74 \mathrm{H} 61 \mathrm{FeN} 2 \mathrm{O} 5$ & 556.695944 & -0.8 & 66.2 & 46.5 & odd \\
\hline & $2+$ & 6 & $\mathrm{C} 75 \mathrm{H} 57 \mathrm{FeN} 6 \mathrm{O}$ & 556.696613 & 0.4 & 75.7 & 51.5 & odd \\
\hline & $2+$ & 7 & $\mathrm{C} 79 \mathrm{H} 61 \mathrm{FeO} 3$ & 556.697956 & 2.8 & 87.6 & 50.5 & odd \\
\hline \multirow[t]{4}{*}{1059.472006} & $1+$ & 1 & $\mathrm{C} 57 \mathrm{H} 67 \mathrm{~N} 6 \mathrm{O} 14$ & 1059.470977 & -1.0 & 32.9 & 28.0 & even \\
\hline & $1+$ & 2 & $\mathrm{C} 61 \mathrm{H} 71016$ & 1059.473663 & 1.6 & 33.7 & 27.0 & even \\
\hline & $1+$ & 3 & $\mathrm{C} 70 \mathrm{H} 59 \mathrm{~N} 8 \mathrm{O} 3$ & 1059.470464 & -1.5 & 70.2 & 46.0 & even \\
\hline & $1+$ & 4 & $\mathrm{C} 74 \mathrm{H} 63 \mathrm{~N} 2 \mathrm{O} 5$ & 1059.473150 & 1.1 & 84.7 & 45.0 & even \\
\hline \multirow{5}{*}{1112.383700} & $1+$ & 1 & $\mathrm{C} 61 \mathrm{H} 68 \mathrm{FeO} 16$ & 1112.385125 & 1.4 & 8.3 & 28.5 & odd \\
\hline & $1+$ & 2 & C57H64FeN6O14 & 1112.382440 & -1.0 & 15.3 & 29.5 & odd \\
\hline & $1+$ & 3 & $\mathrm{C} 70 \mathrm{H} 56 \mathrm{FeN} 8 \mathrm{O} 3$ & 1112.381927 & -1.4 & 50.1 & 47.5 & odd \\
\hline & $1+$ & 4 & $\mathrm{C} 74 \mathrm{H} 60 \mathrm{FeN} 2 \mathrm{O} 5$ & 1112.384612 & 1.0 & 61.5 & 46.5 & odd \\
\hline & $1+$ & 5 & $\mathrm{C} 75 \mathrm{H} 56 \mathrm{FeN} 6 \mathrm{O}$ & 1112.385949 & -889.9 & 663.5 & 51.5 & odd \\
\hline
\end{tabular}

Figure $\mathbf{S 6}$ : HMRS of compound La $\mathbf{L}^{4}$. Peak at 338 is due to the presence of erucamide in Eppendorf's.

$\mathrm{mV}$

Chromatogram(NH40f4-analytique-UBO.Icd)

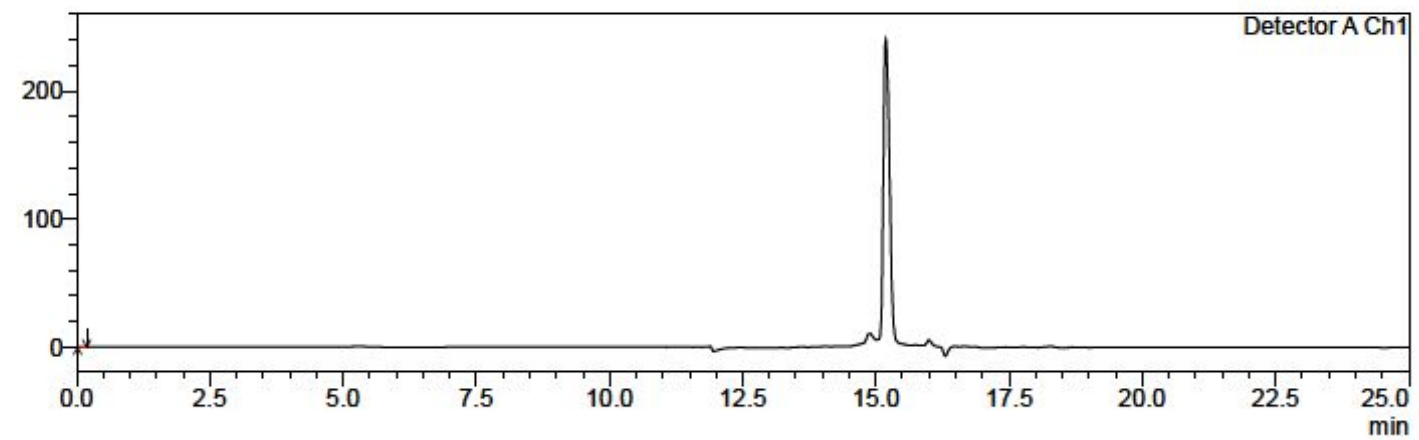

Figure S7 : Analytical HPLC of the [EuL ${ }^{4 a}$ ] complex. Column: Vision HT C18 HL $5 \mu 250 \times 4.6 \mathrm{~mm}$. Gradient: 100\% $\mathrm{H}_{2} \mathrm{O} 0-5 \mathrm{~min}, 0-90 \%$ ACN 5$15 \mathrm{~min}, 90 \% \mathrm{ACN} 15-20 \mathrm{~min}, 100 \% \mathrm{H}_{2} \mathrm{O} 20-25 \mathrm{~min}$. Flow: $1 \mathrm{~mL} / \mathrm{min}$. retention time $=15.186 \mathrm{~min}$. 

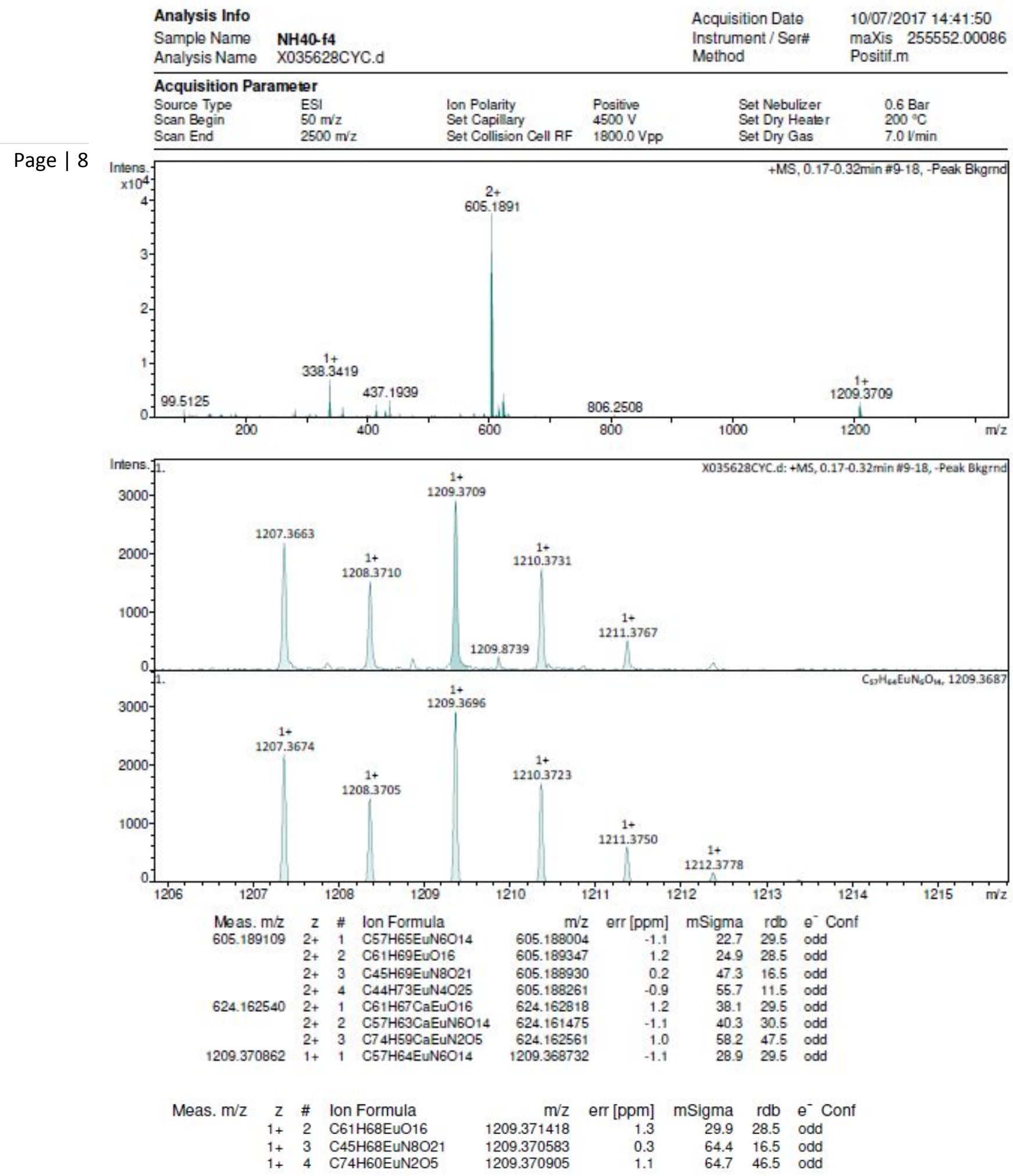

Figure S8: HMRS of compound [EuL ${ }^{4 a}$ ]. Peak at 338 is due to the presence of erucamide in Eppendorf's. 
$\mathrm{mV}$

Chromatogram(NH65-phaseorga-UBO.Icd)

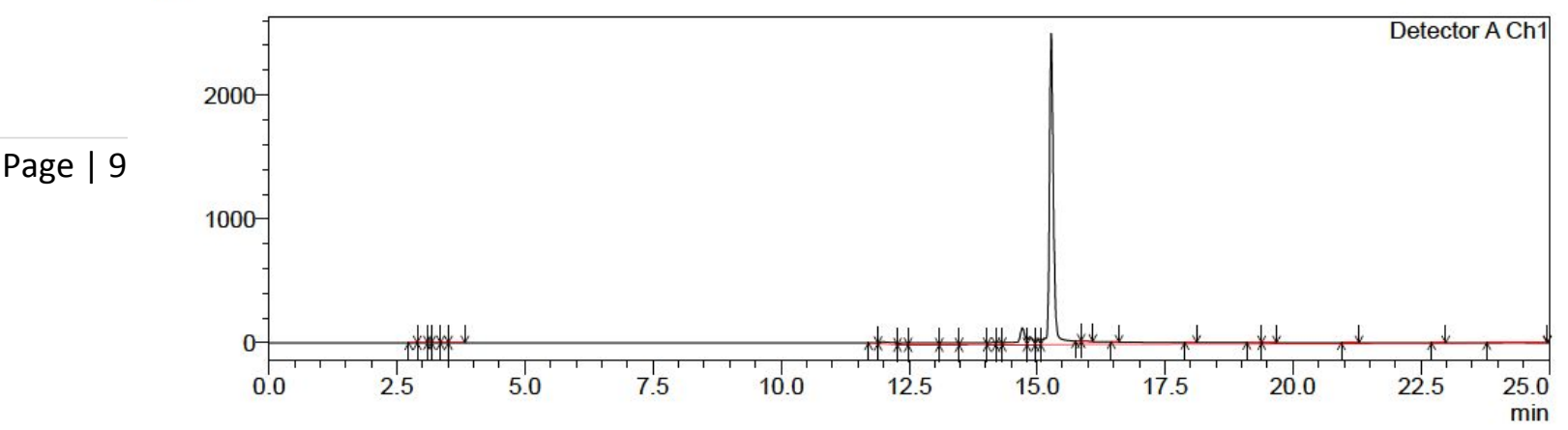

Figure S9 : Analytical HPLC of the [SmL ${ }^{4 a}$ ] complex. Column: Vision HT C18 HL $5 \mu 250 \times 4.6$ mm. Gradient: 100\% $\mathrm{H}_{2} \mathrm{O} 0-5$ $\mathrm{min}, 0-90 \%$ ACN 5-15 min, $90 \%$ ACN 15-20 min, 100\% H $\mathrm{H}_{2}$ 20-25 min. Flow : $1 \mathrm{~mL} / \mathrm{min}$. retention time $=15.278 \mathrm{~min}$. 
Analysis Info

Sample Name

$\begin{array}{ll}\text { Sample Name } & \text { NH65-phase MeOH } \\ \text { Analysis Name } & \text { X035629CYC.d }\end{array}$

Acquisition Parameter

Source Type

Scan Begin

Scan End

$\mathrm{ESI}$
$50 \mathrm{~m} / \mathrm{z}$

$2500 \mathrm{~m} / \mathrm{z}$
Acquisition Date

Instrument / Ser\# Method
10/07/2017 14:43:17

maXis 255552.00086 Positif.m

Page | 10
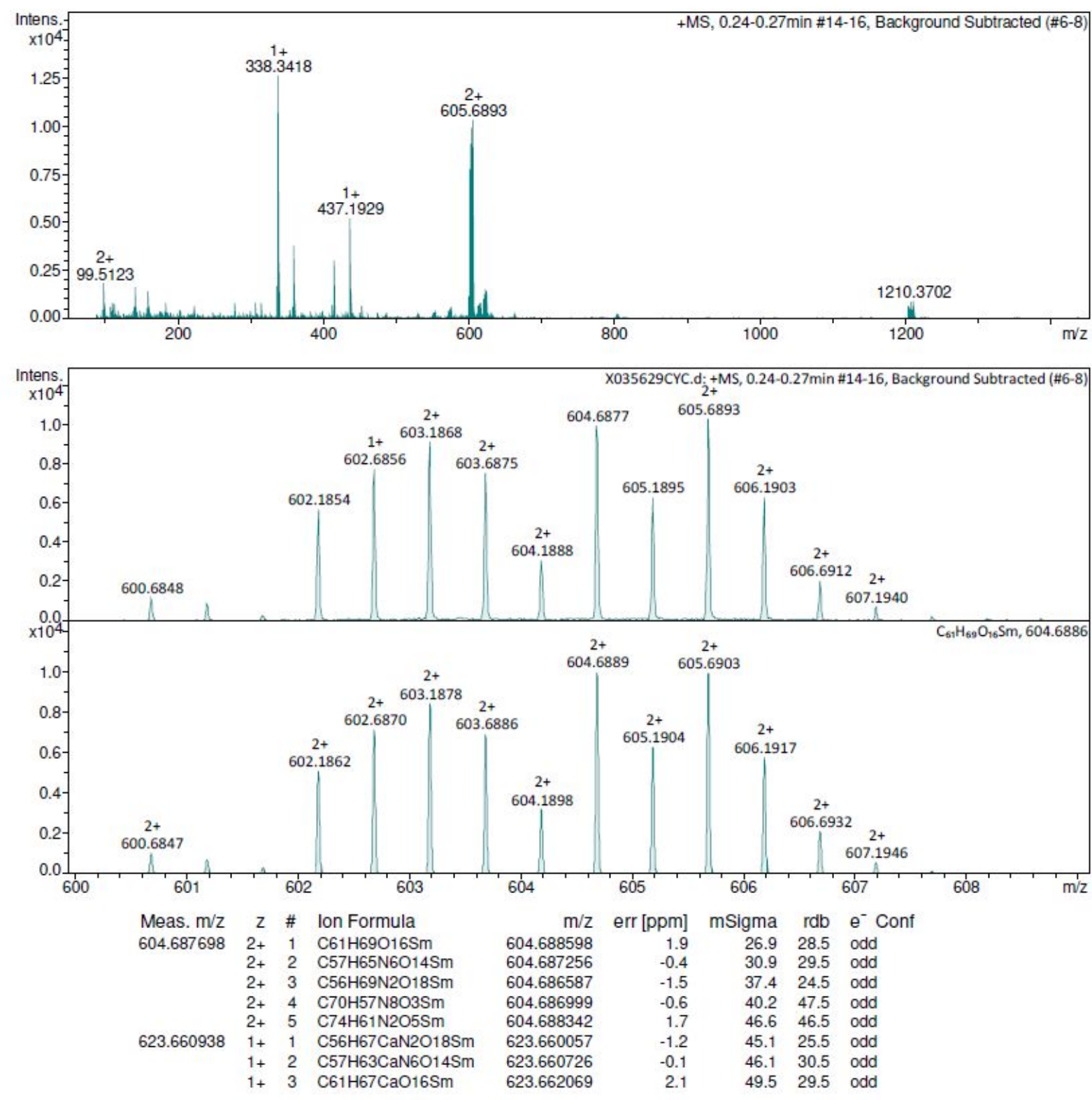

$\begin{array}{rrrrl}\mathrm{m} / \mathrm{z} & \text { err [ppm] } & \text { mSigma } & \text { rdb } & \mathrm{e}^{-} \text {Conf } \\ 623.661651 & 1.2 & 59.0 & 17.5 & \text { odd } \\ 623.660983 & 0.2 & 65.1 & 12.5 & \text { odd } \\ 623.660314 & -0.9 & 71.7 & 7.5 & \text { odd } \\ 1208.369085 & 0.4 & 85.4 & 16.5 & \text { odd } \\ 1208.367748 & -0.7 & 87.7 & 11.5 & \text { odd } \\ 1208.367235 & -1.0 & 90.8 & 29.5 & \text { odd } \\ 1208.366410 & -1.8 & 91.1 & 6.5 & \text { odd } \\ 1208.369920 & 1.3 & 96.4 & 28.5 & \text { odd }\end{array}$

Figure S10 : HMRS of compound [SmL ${ }^{4 a}$ ]. Peak at 338 is due to the presence of erucamide in Eppendorf's. 
Page | 11
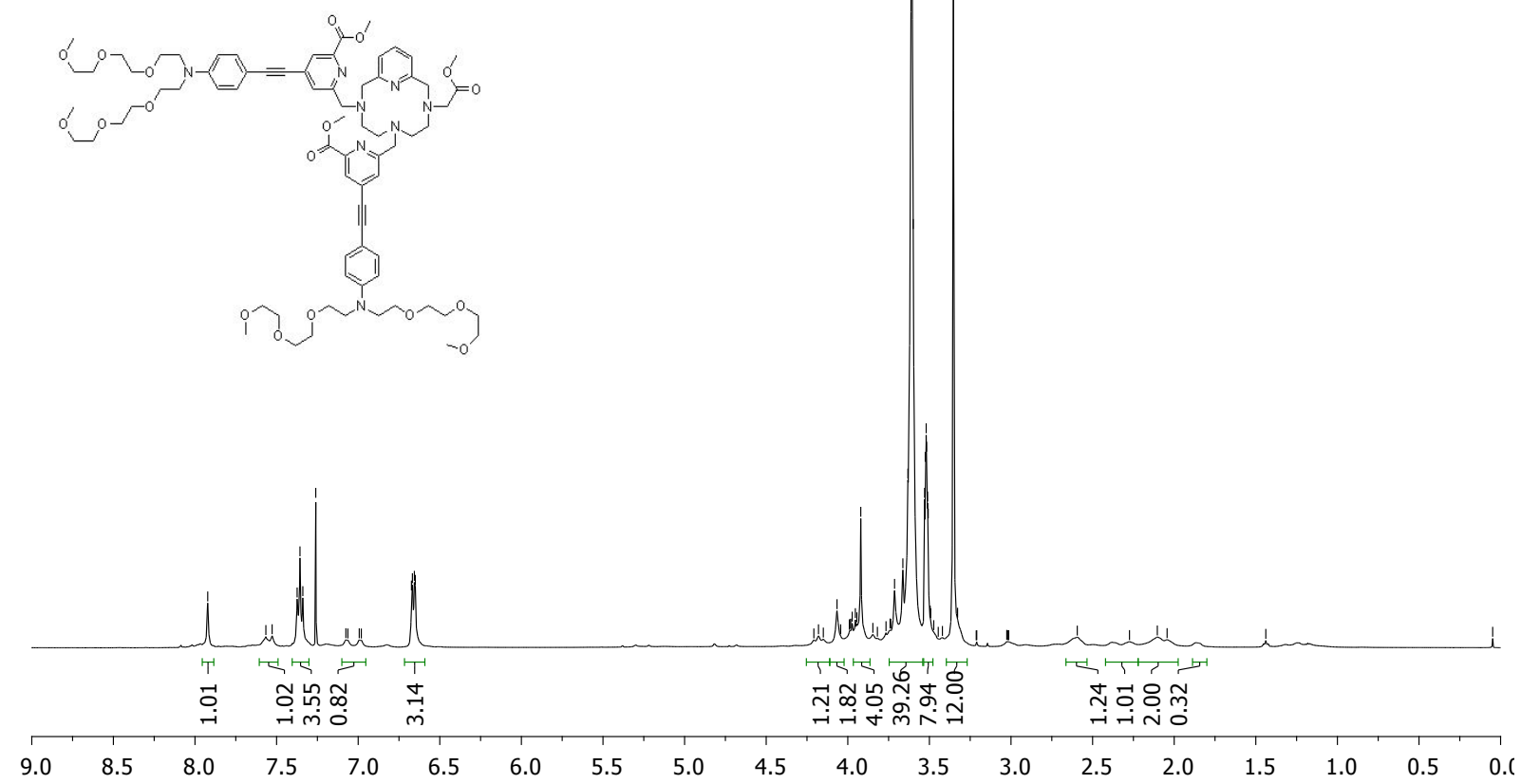

Figure S11 : ${ }^{1} \mathrm{H}$ NMR of compound 5.

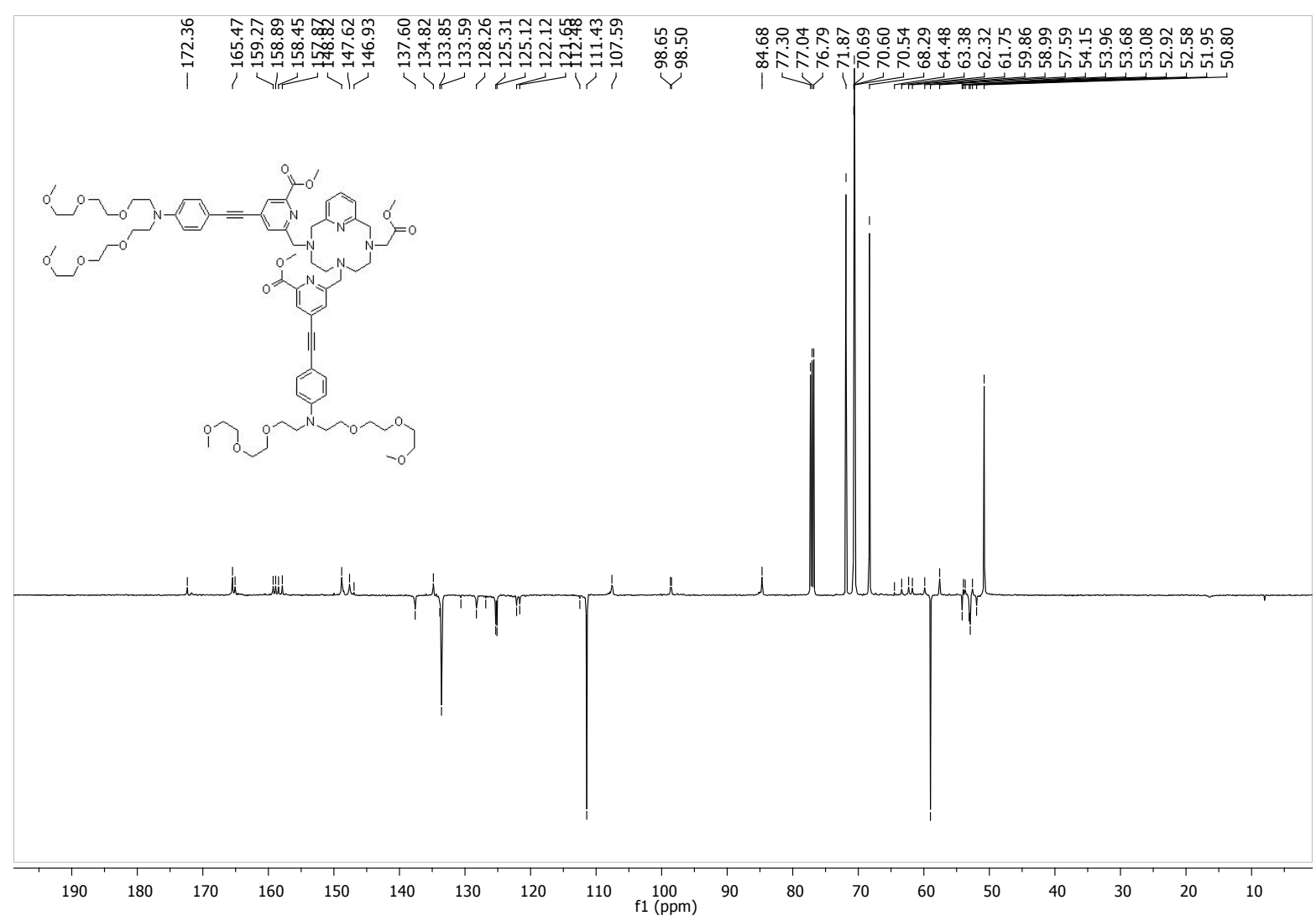

Figure S12: ${ }^{13} \mathrm{C}$ NMR of compound 5. 

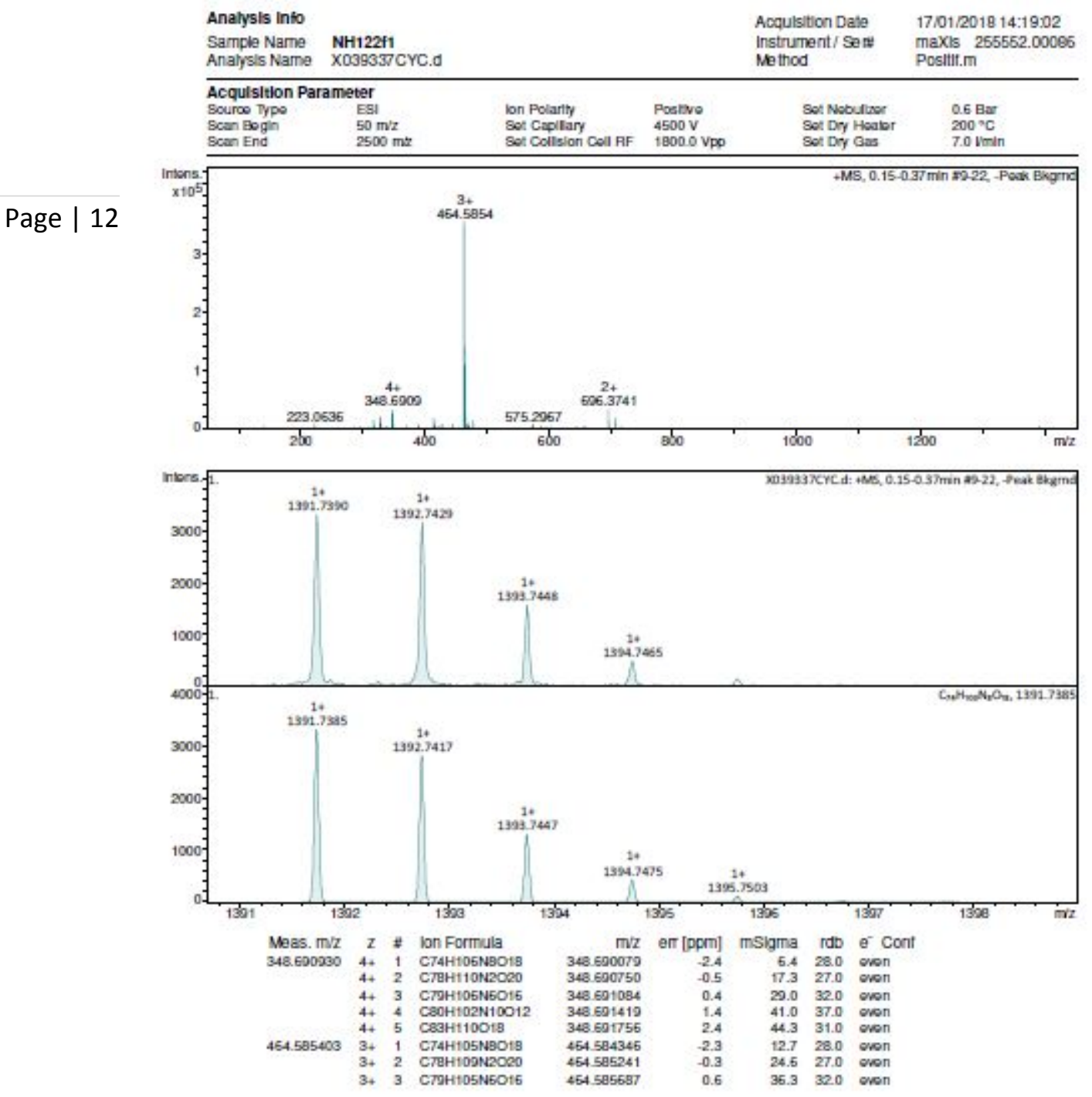

\begin{tabular}{|c|c|c|c|c|c|c|c|c|}
\hline \multirow[t]{3}{*}{ Meas. $m / z$} & $z$ & $\#$ & Ion Formula & $\mathrm{m} / 2$ & ert [ppm] & msigma & $\mathrm{rdb}$ & \\
\hline & $3+$ & 4 & C8OH101N10O12 & 454.586133 & 1.6 & 46.2 & 37.0 & \\
\hline & 3. & 5 & Сеан102018 & 454.586582 & 2.5 & 51.3 & 31.0 & \\
\hline \multirow{3}{*}{696.374005} & $2+$ & 1 & C74H104NBO18 & 696.372881 & -1.7 & 6.3 & 28.0 & \\
\hline & $2+$ & 2 & C7RHIOSN2OEO & 695.314223 & 0.2 & 12.6 & 27.0 & \\
\hline & $2+$ & 3 & C79HIOLNEOTE & 695.3749022 & 1.2 & 31.4 & 320 & \\
\hline \multirow[t]{3}{*}{707.364968} & $2+$ & 1 & C7RH107N2NaO20 & 707.365125 & & 8.1 & 27.0 & \\
\hline & $2+$ & 2 & $\mathrm{crot}$ & & 1 & 14.6 & 0 & \\
\hline & $2+$ & 3 & C74H103N4BNaOIS & 707363853 & -1.4 & 14.8 & 28.0 & \\
\hline \multirow[t]{3}{*}{1301.739955} & $1+$ & 1 & $\mathrm{C} 9 \mathrm{OH} 103 \mathrm{O} 13$ & & 0.3 & 31.8 & 40.0 & \\
\hline & $1+$ & 2 & $\mathrm{CTH}$ & 70 & 1.6 & 51.6 & 27.0 & \\
\hline & $1+$ & 3 & $\mathrm{C} 74 \mathrm{H}$ & & -0.3 & 5.2 & 28.0 & \\
\hline \multirow{2}{*}{413.721363} & $1+$ & 1 & C74H102N4BNSO18 & 1413.720429 & -0.7 & 61.4 & 28.0 & \\
\hline & $1+$ & 2 & C7RHIOSN2N & 1413.723115 & 1.2 & 71.5 & 27.0 & \\
\hline
\end{tabular}

Figure S13 : HRMS of compound 5. 
Page | 13

$\mathrm{mV}$

Chromatogram(NH130f1-analytique-UBO.Icd)

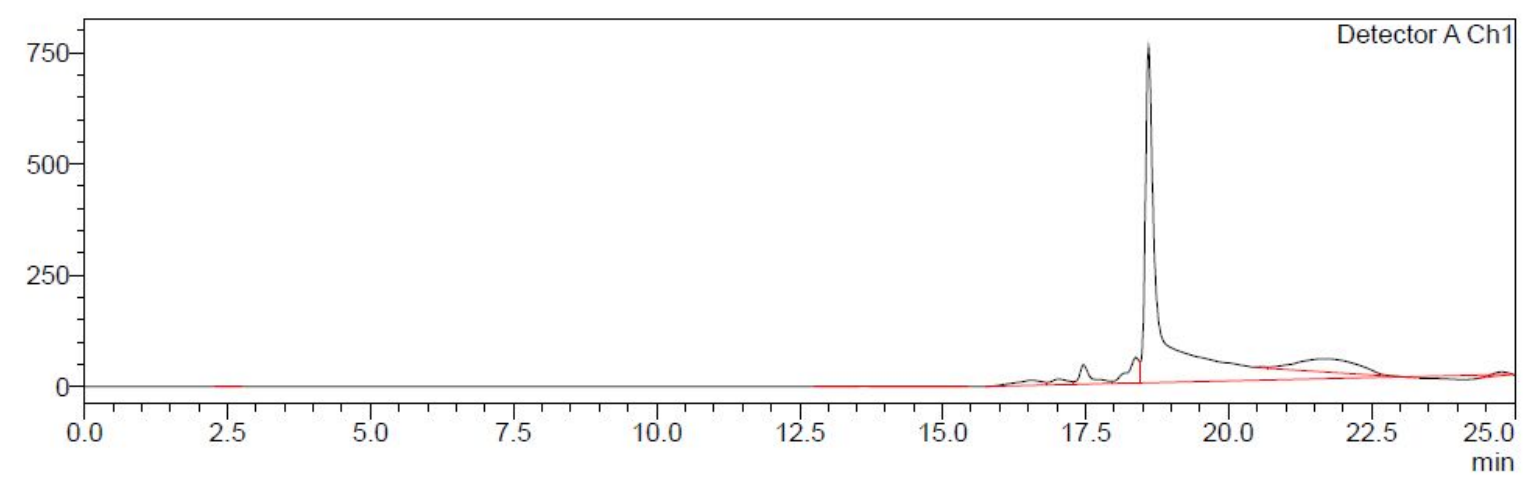

Figure S14 : Analytical HPLC of compound L4b. Column: Vision HT C18 HL $5 \mu 250 \times 4.6 \mathrm{~mm}$. Gradient: 100\% $\mathrm{H}_{2} \mathrm{O} 0-5 \mathrm{~min}, 0-$ $90 \% \mathrm{MeOH}$ 5-15 min, 90\% MeOH 15-20 min, 100\% H $\mathrm{H}_{2}$ 20-25min. Flow: 1mL/min. retention time $=18.601 \mathrm{~min}$.

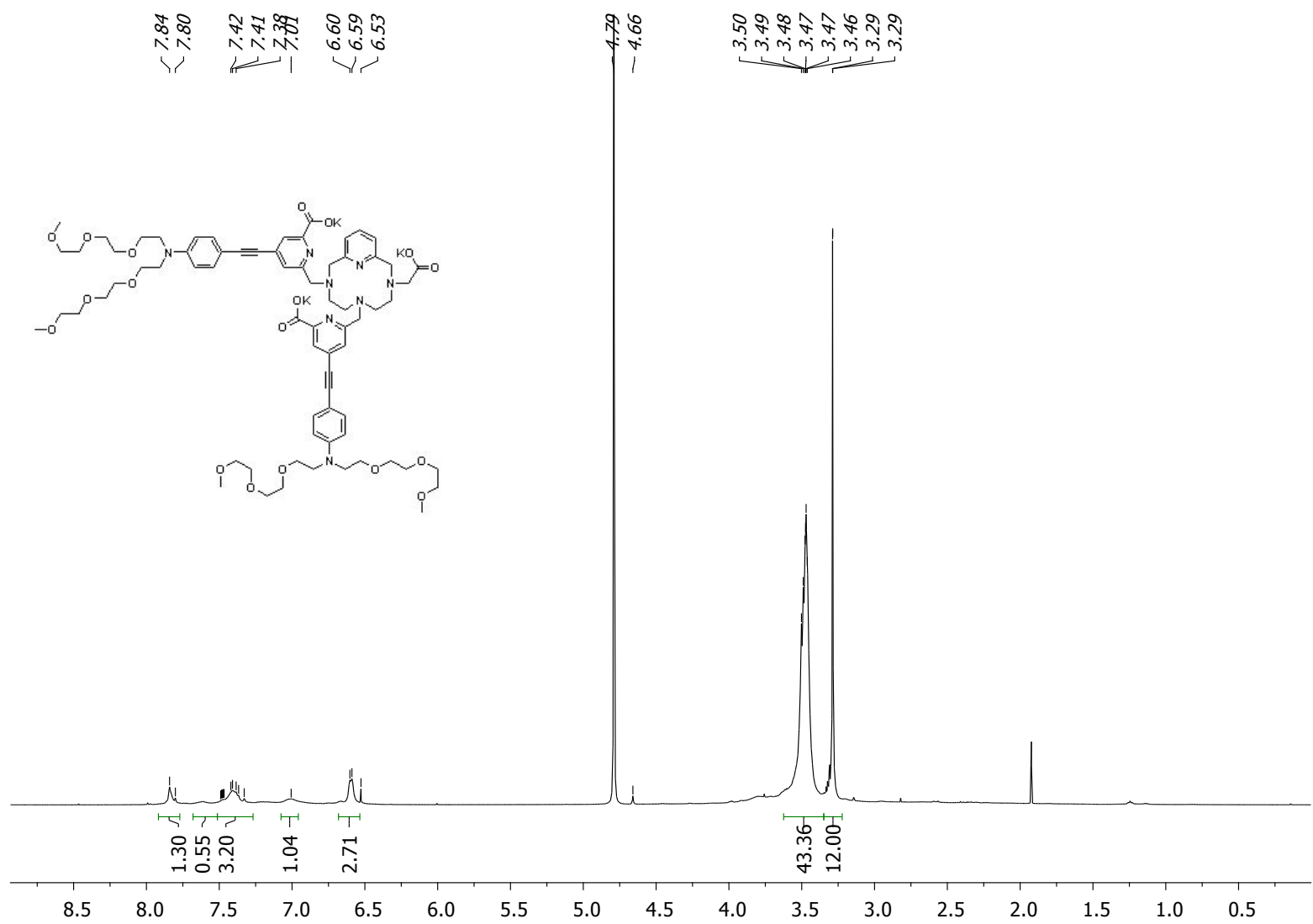

Figure S15: ${ }^{1} \mathrm{H}$ NMR of compound $\mathrm{L}^{4 b}$. 


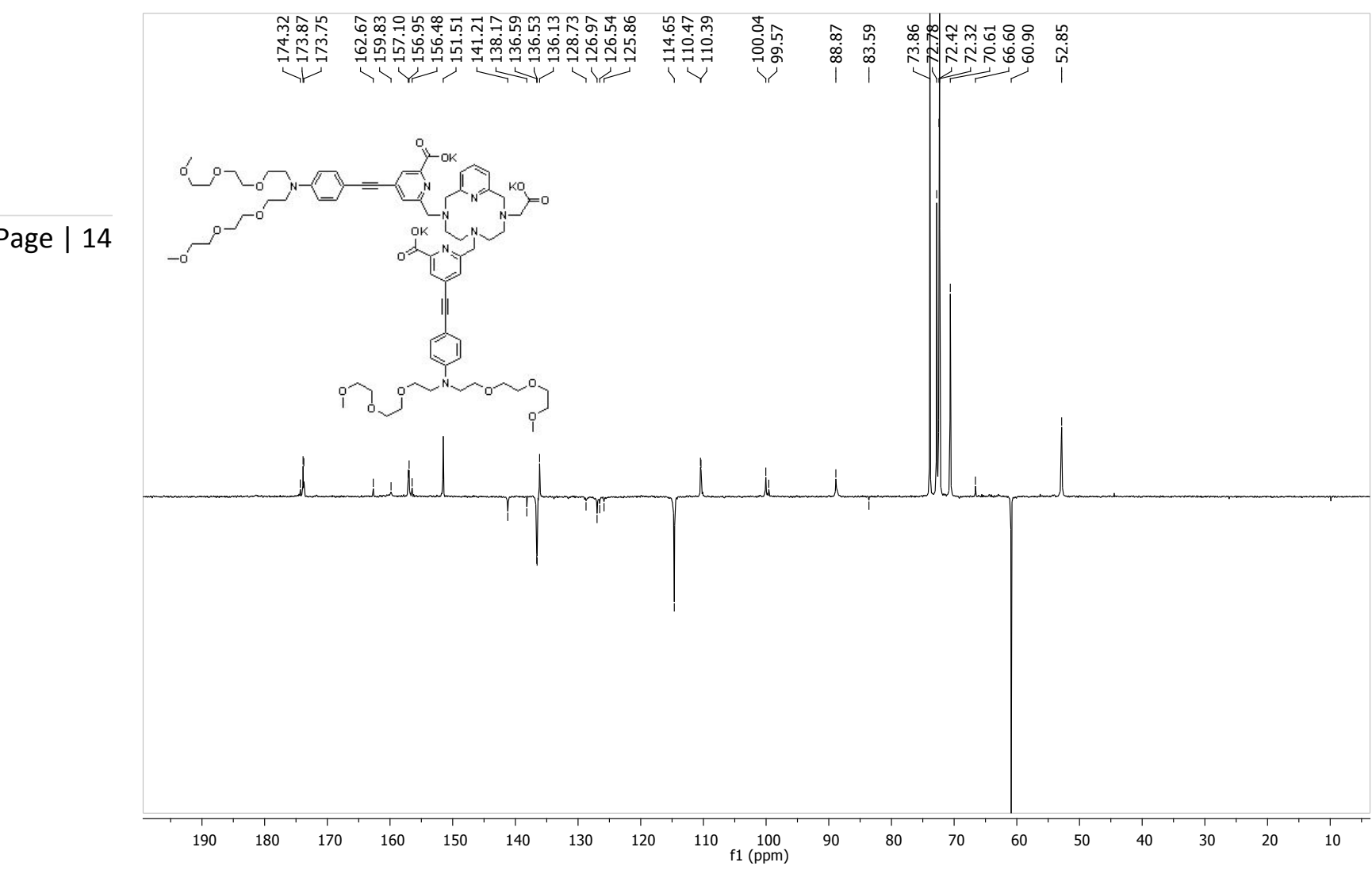

Figure S16 : ${ }^{13} \mathrm{C}$ NMR of compound $\mathrm{L}^{4 b}$. 

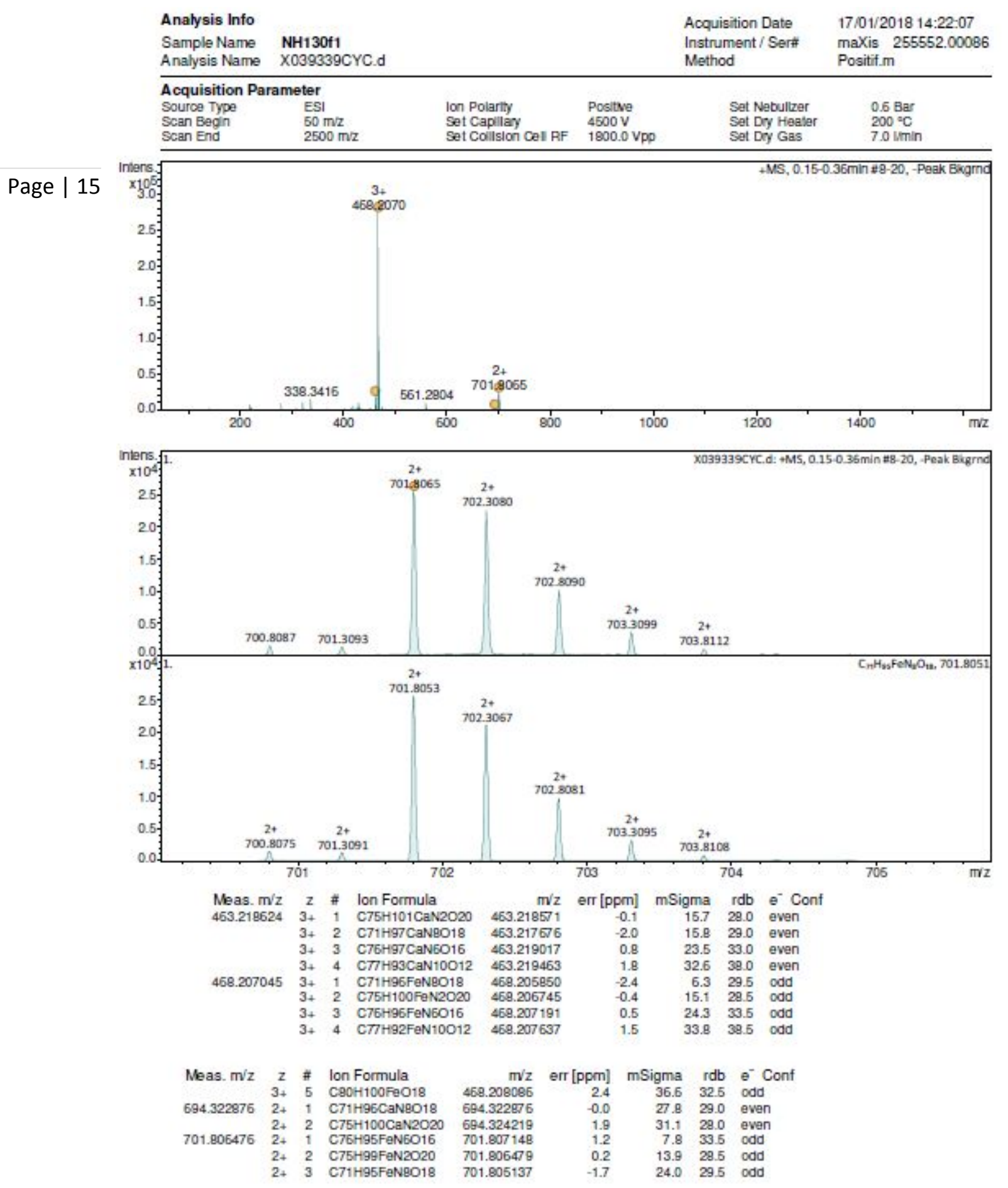

Figure S17 : HRMS of compound $\mathbf{L}^{4 b}$. Peak at 338 is due to the presence of erucamide in the Eppendorf. 


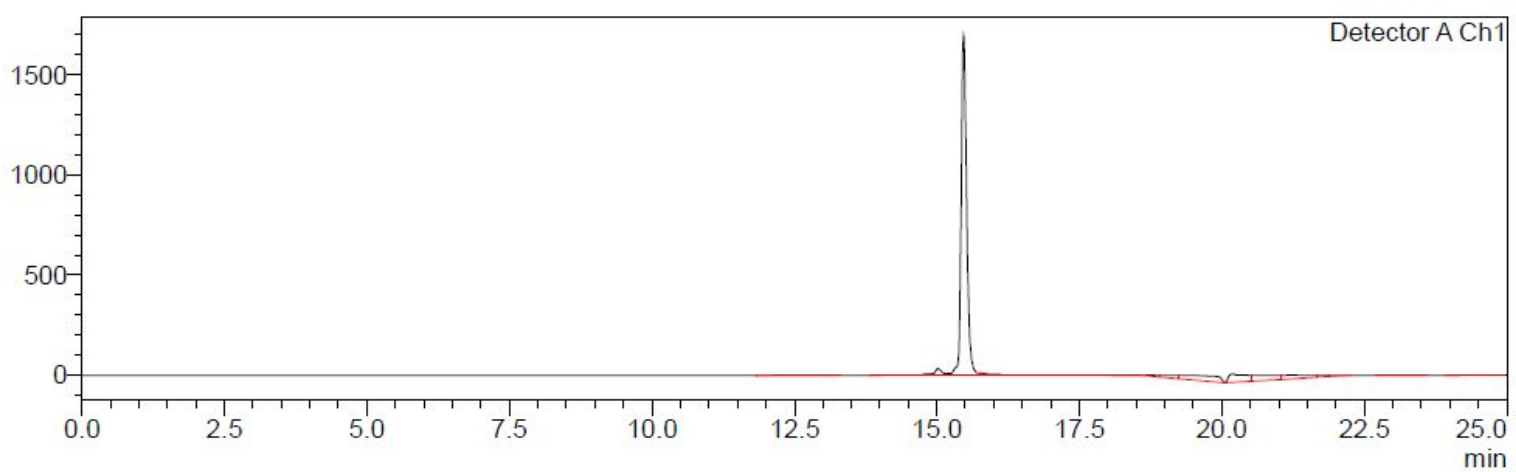

Figure S18 : Analytical HPLC of the [YbL ${ }^{4 b}$ ] complex. Column: Vision HT C18 HL $5 \mu 250 \times 4.6 \mathrm{~mm}$. Gradient: 100\% $\mathrm{H}_{2} \mathrm{O} 0-5 \mathrm{~min}$, 0-90\% ACN 5-15 min, 90\% ACN 15-20 min, 100\% $\mathrm{H}_{2} \mathrm{O} 20-25 \mathrm{~min}$. Flow: $1 \mathrm{~mL} / \mathrm{min}$. retention time $=15.469 \mathrm{~min}$.
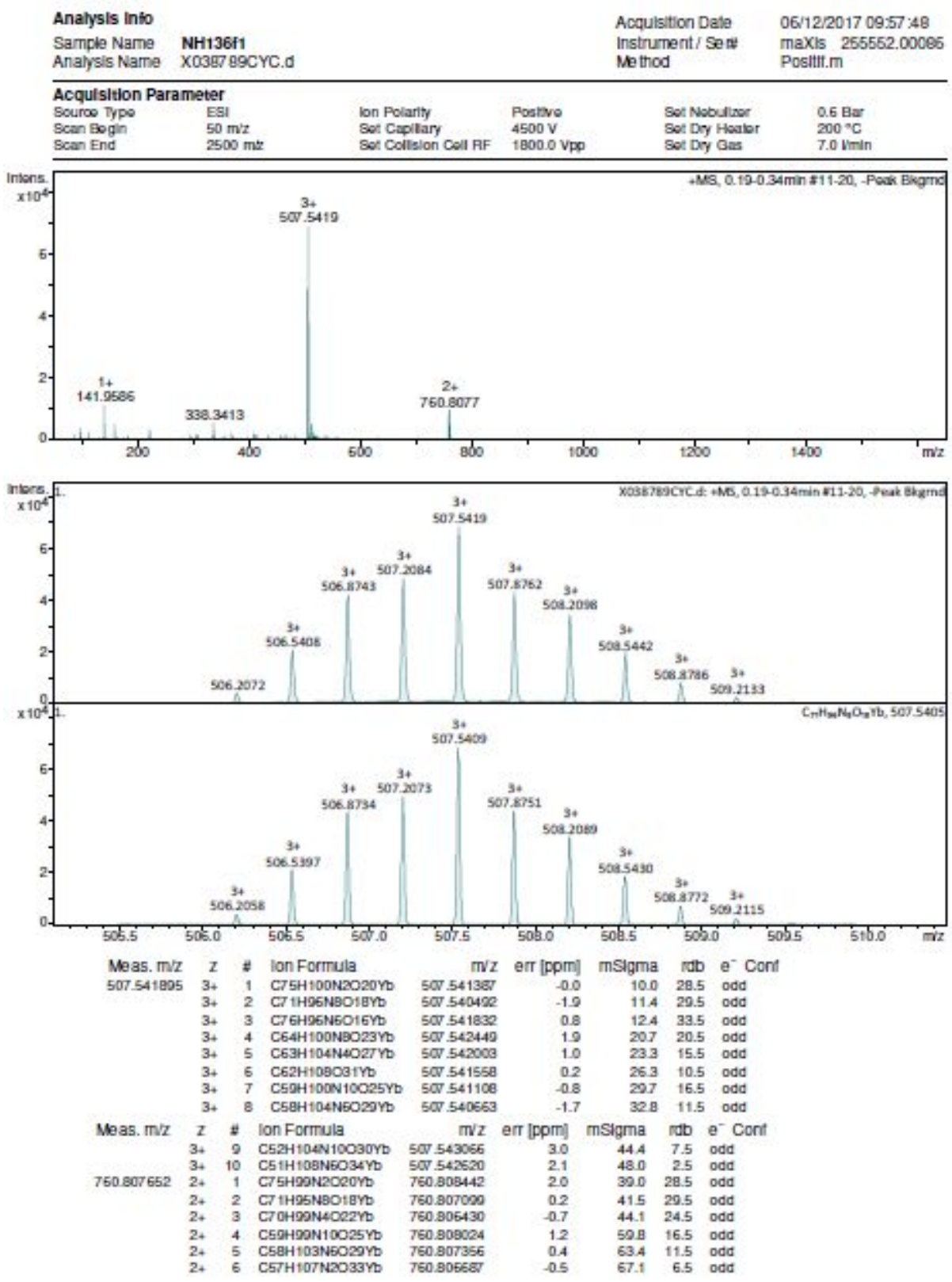

Figure S19 : HRMS of the $\left[\mathrm{YbL}^{4 b}\right]$ complex. Peak at 338 is due to the presence of erucamide in the Eppendorf. 
Page | 17

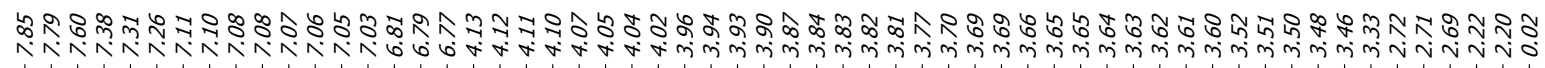

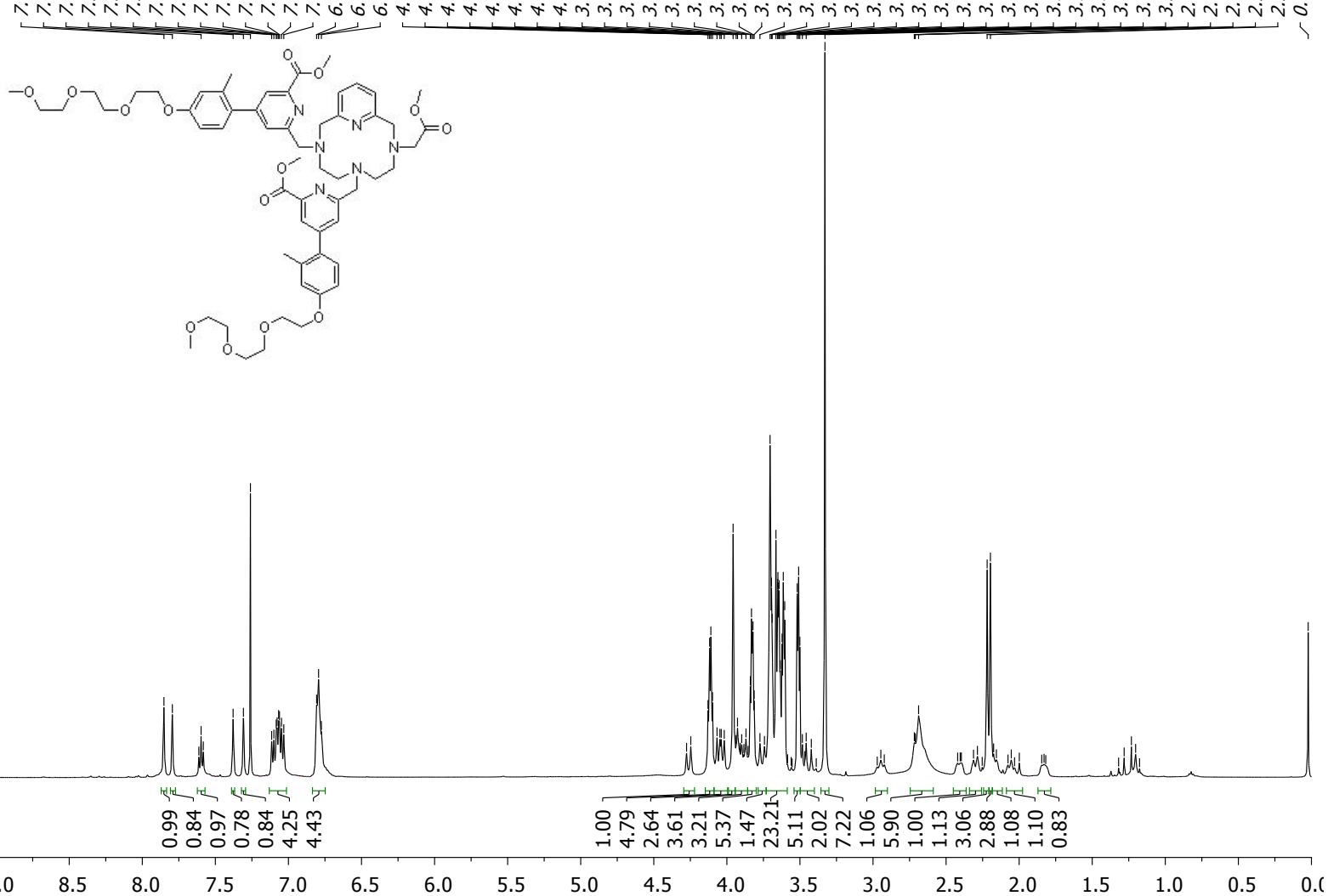

Figure S20 : ${ }^{1} \mathrm{H}$ NMR of compound 7.

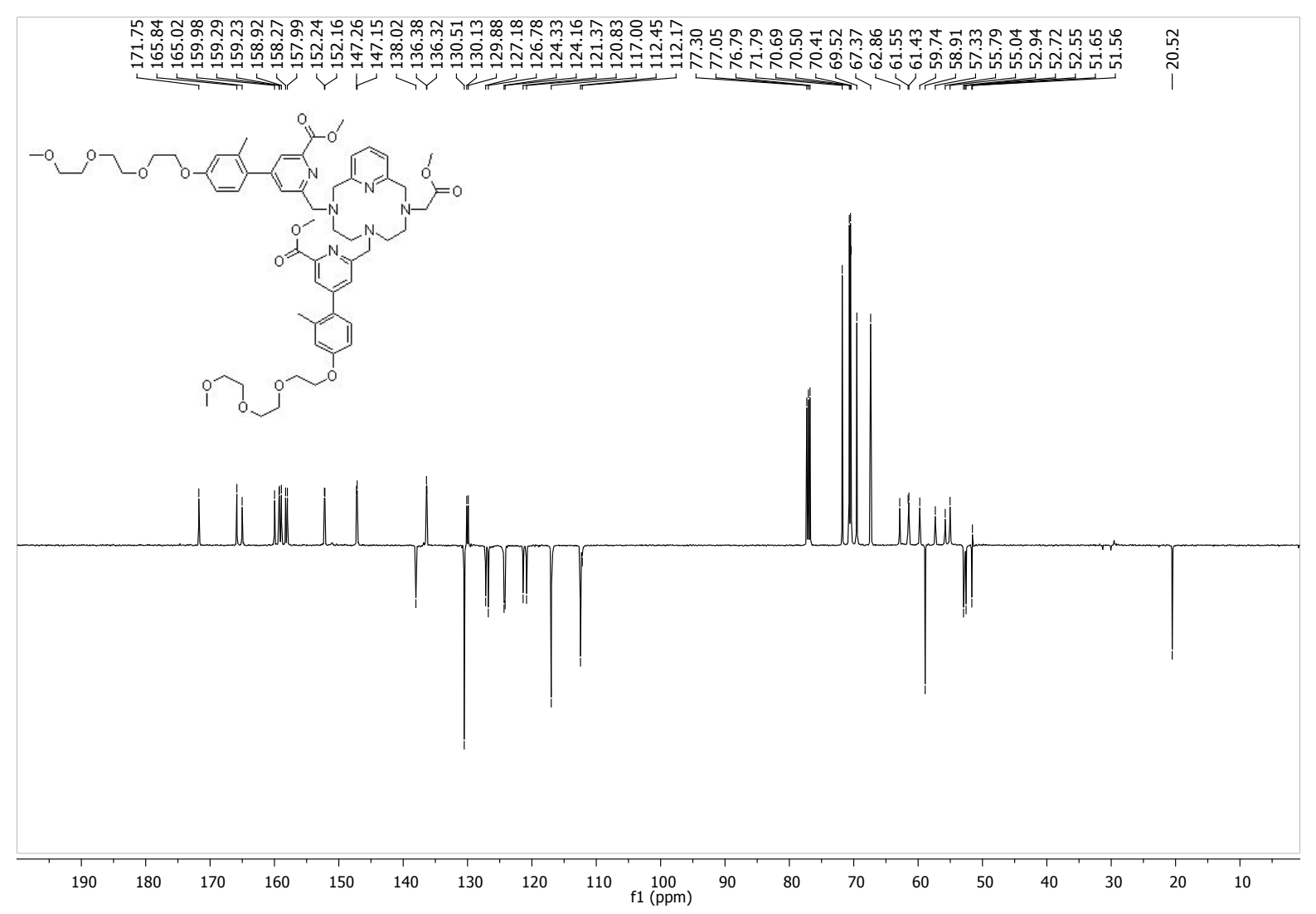

Figure S21: ${ }^{13} \mathrm{C}$ NMR of compound 7. 


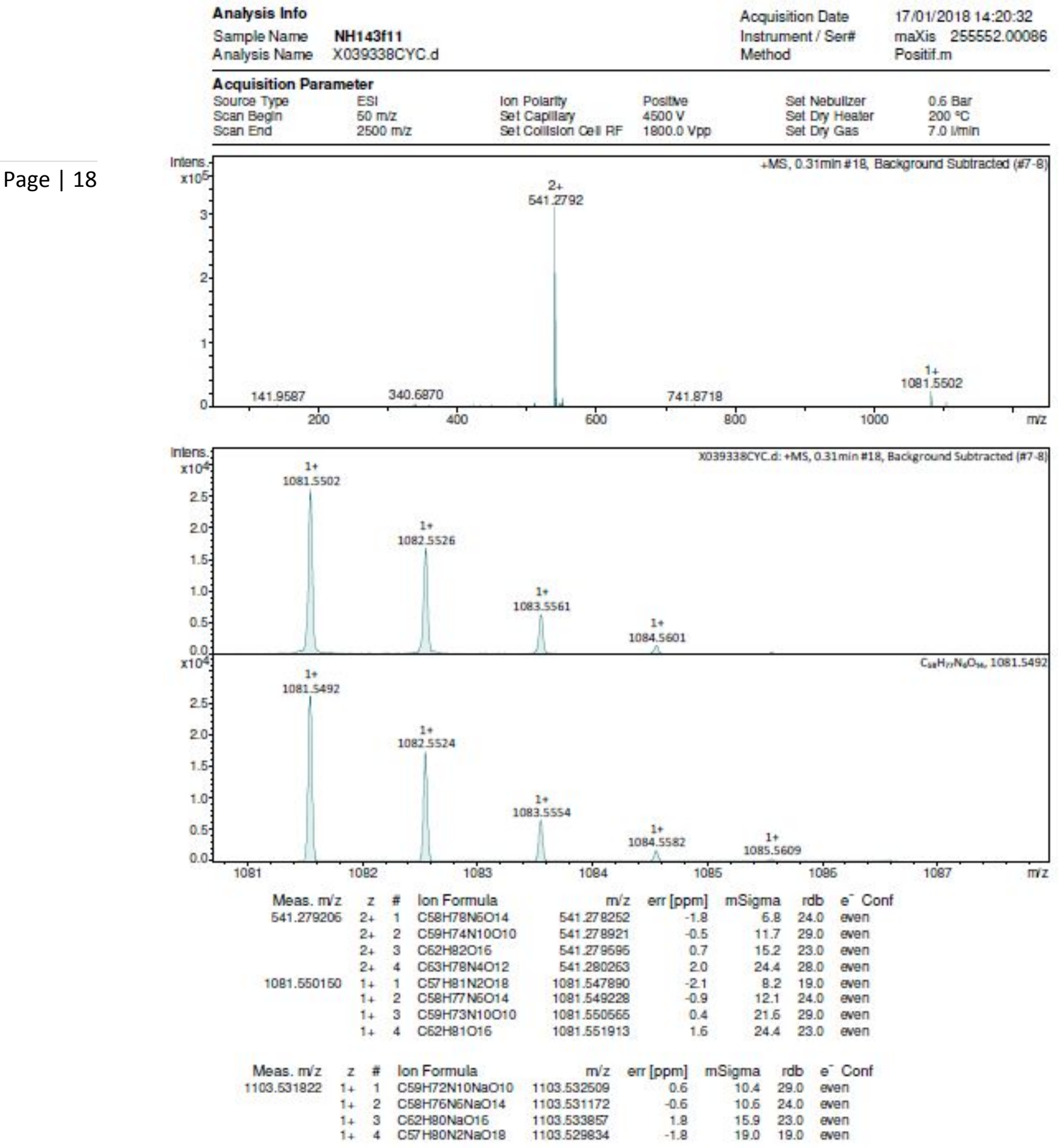

Figure S22 : HRMS of compound 7. 
$\mathrm{mV}$

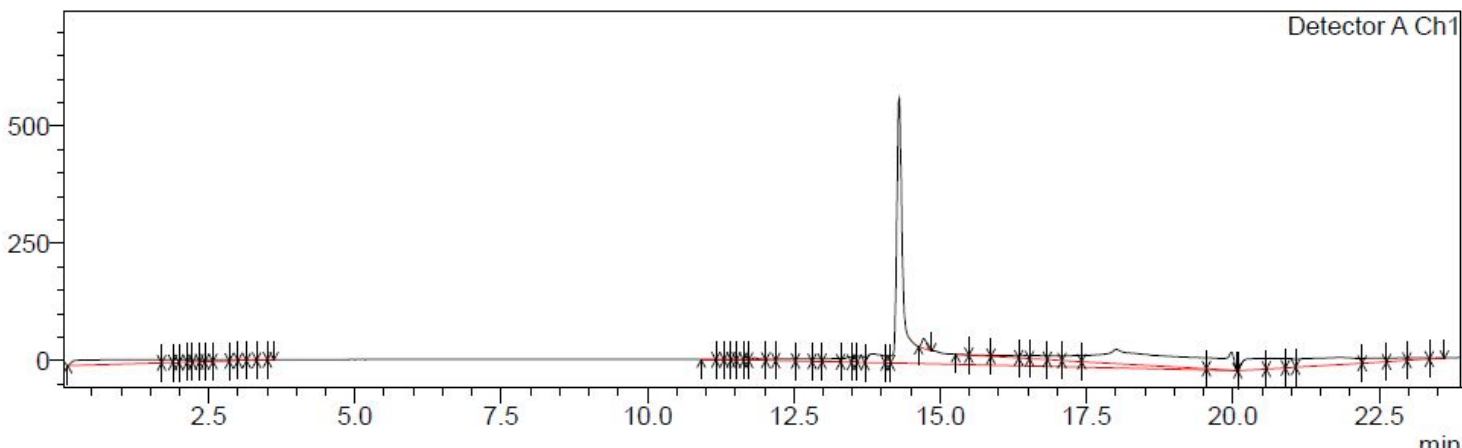

Figure S23 : Analytical HPLC of compound L4c. Column: Vision HT C18 HL $5 \mu 250 \times 4.6 \mathrm{~mm}$. Gradient: $100 \% \mathrm{H}_{2} \mathrm{O} 0-5 \mathrm{~min}, 0-$ 90\% ACN 5-15 min, 90\% ACN 15-20 min, 100\% $\mathrm{H}_{2} \mathrm{O}$ 20-25min. Flow : 1mL/min. retention time $=14.298 \mathrm{~min}$.

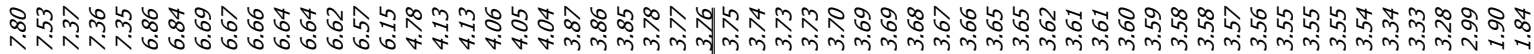
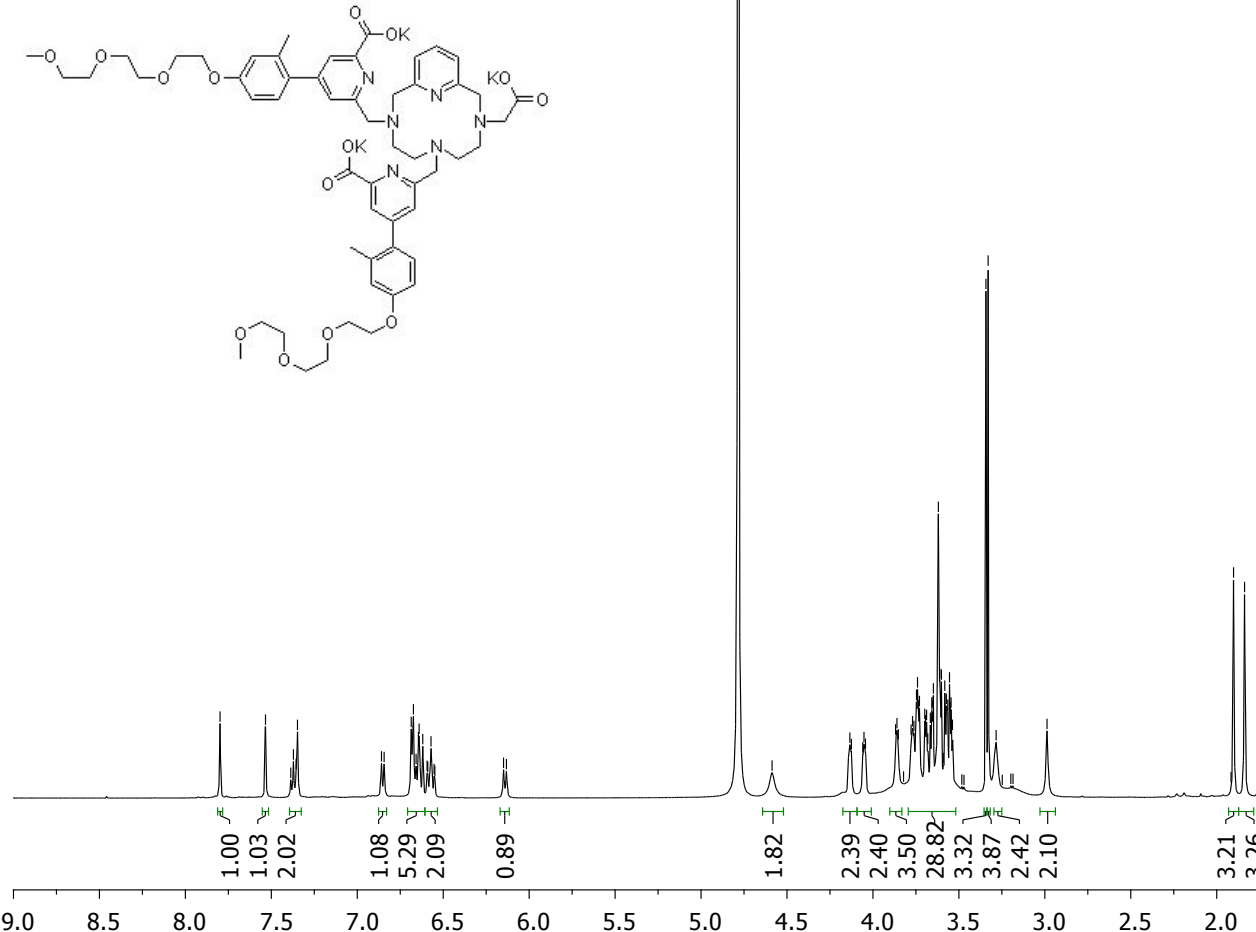

익

9.0

Figure S24 : ${ }^{1} \mathrm{H}$ NMR of compound $\mathrm{L}^{4 \mathrm{c}}$. 


\section{Page | 20}

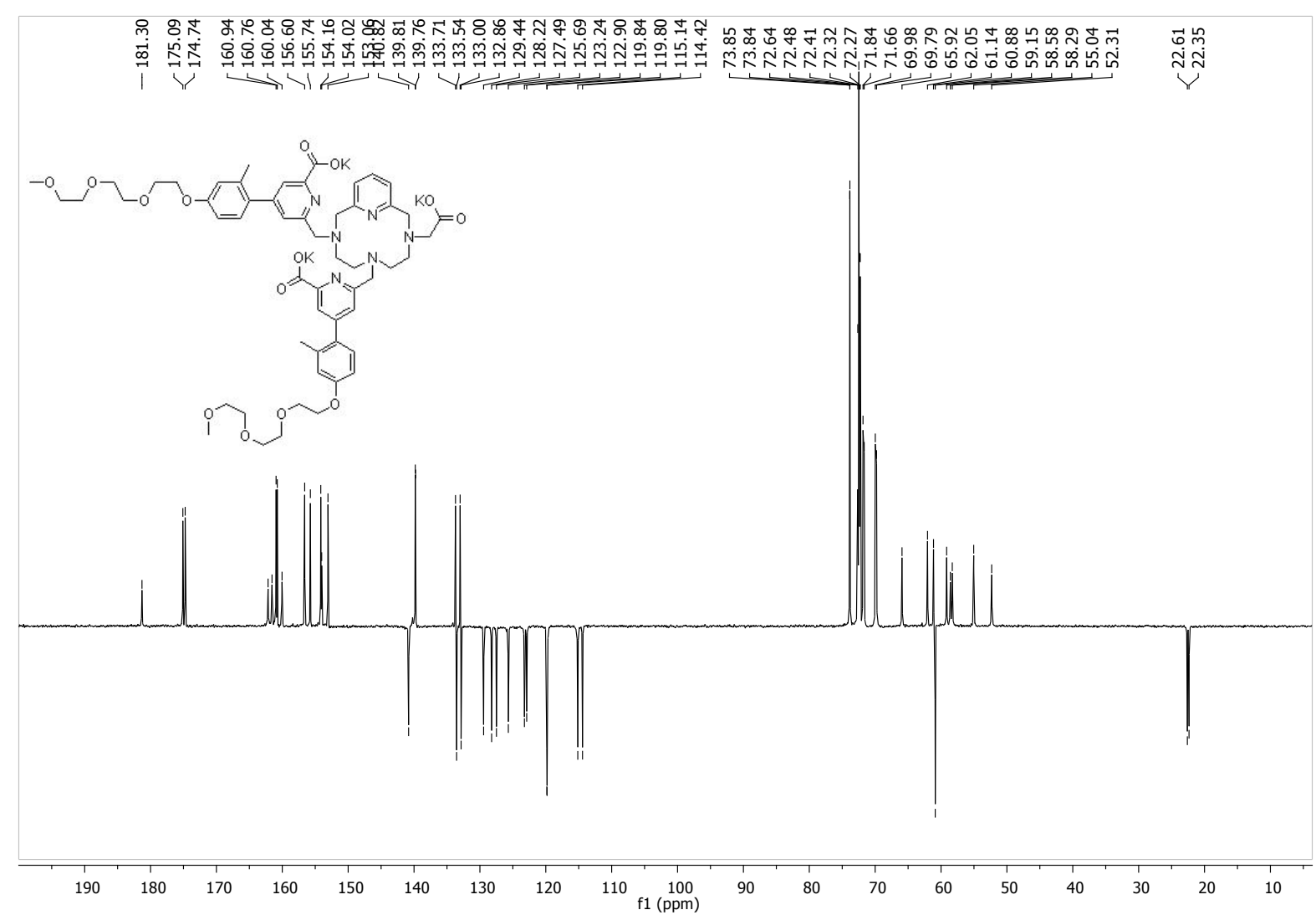

Figure S25: ${ }^{13} \mathrm{C}$ NMR of compound $\mathrm{L}^{4 \mathrm{c}}$. 

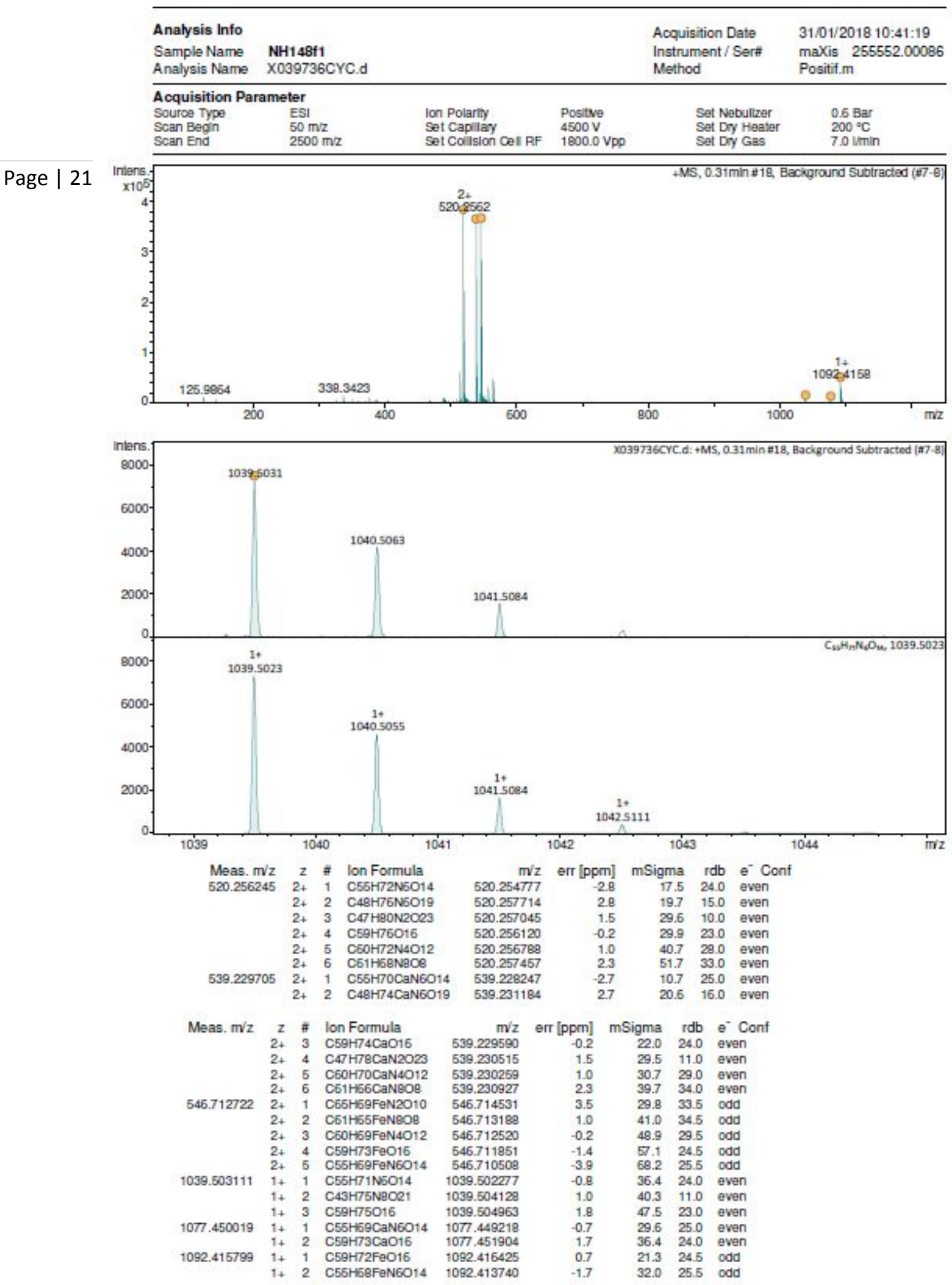

Figure S26 : HRMS of compound $\mathrm{L}^{4 c}$. 
$\mathrm{mV}$

Chromatogram(NH152f1-analytique-UBO.Icd)

Page | 22

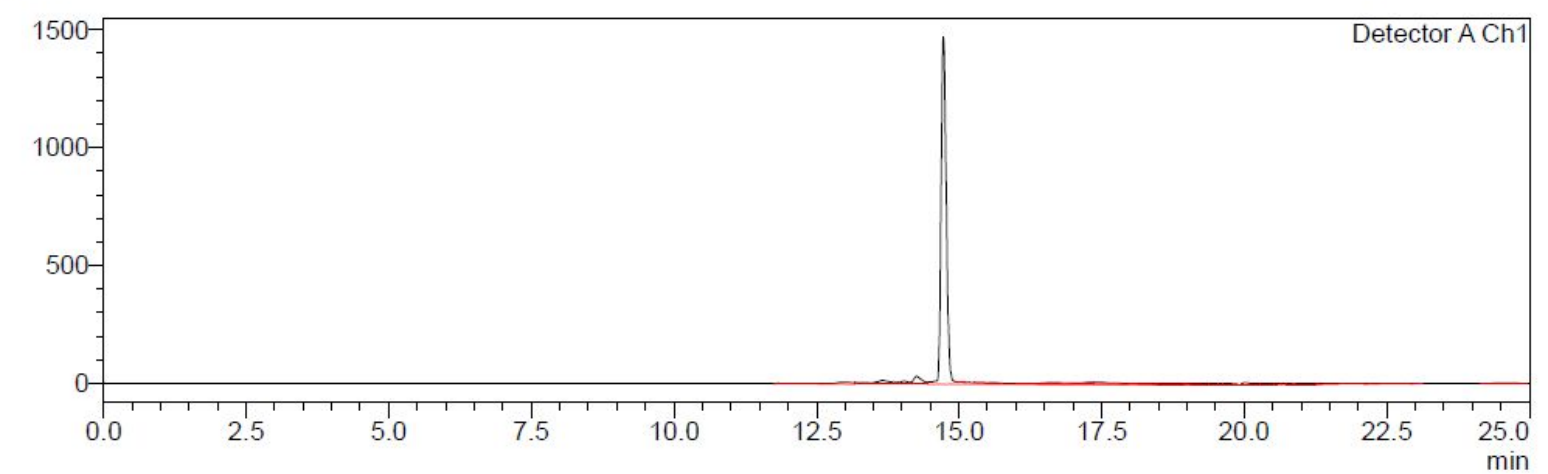

Figure S27 : Analytical HPLC of the [TbL $\left.{ }^{4 c}\right]$ complex. Column: Vision HT C18 HL $5 \mu 250 \times 4.6 \mathrm{~mm}$. Gradient: 100\% $\mathrm{H}_{2} \mathrm{O} 0-5 \mathrm{~min}$, 0-90\% ACN 5-15 min, 90\% ACN 15-20 min, 100\% $\mathrm{H}_{2} \mathrm{O}$ 20-25min. Flow: 1mL/min. retention time = $14.725 \mathrm{~min}$. 


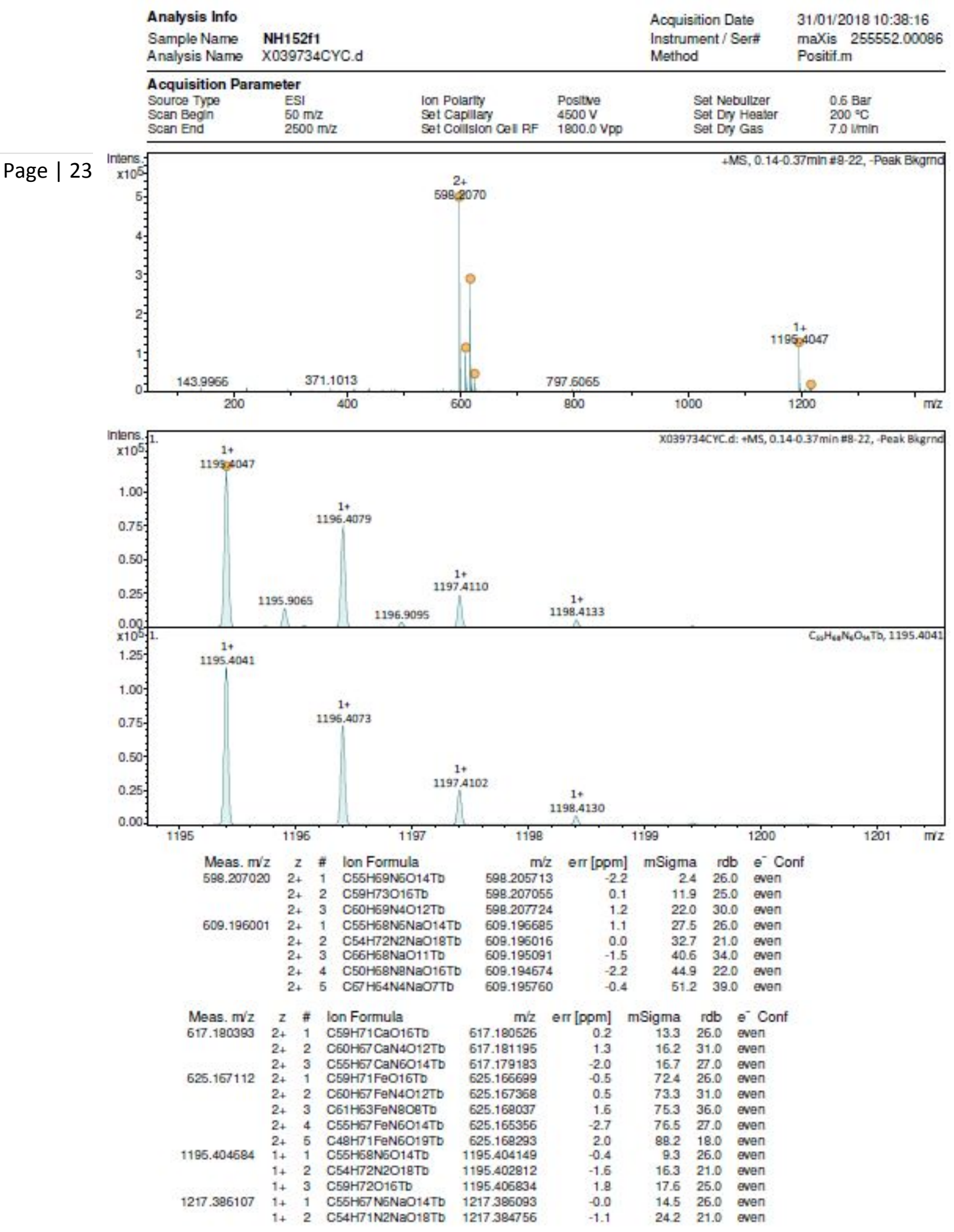

Figure S28 : HRMS of the $\left[\mathrm{TbL}^{4 \mathrm{c}}\right]$ complex. 
$\mathrm{mV}$

Chromatogram(NH153f1-analytique-UBO.Icd)

Page | 24

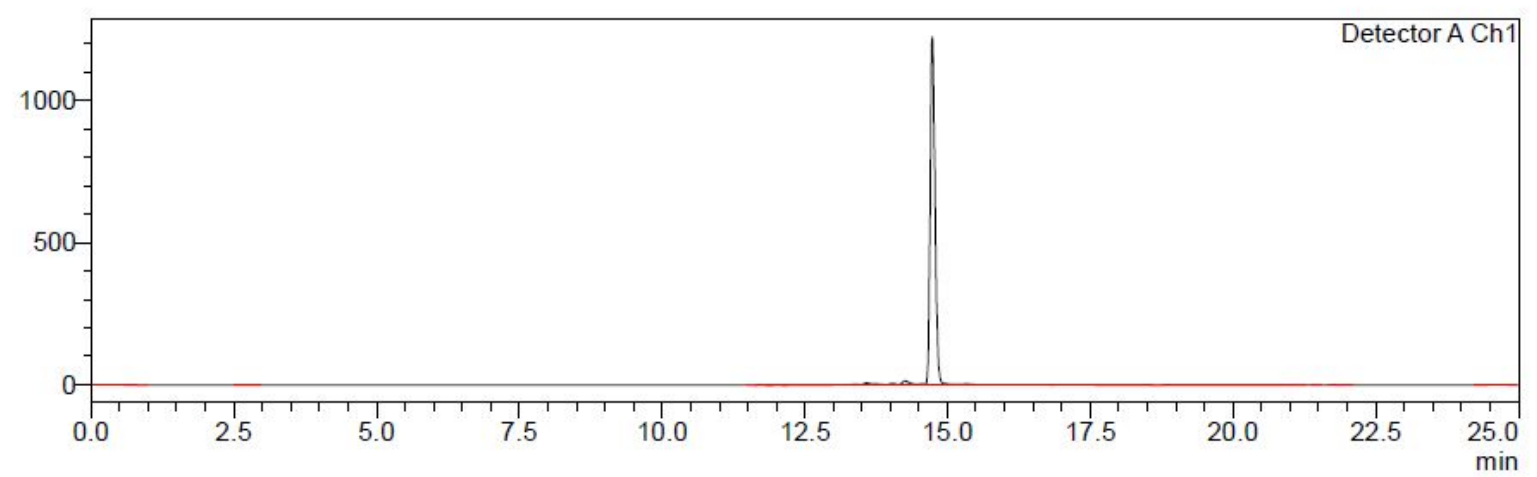

Figure S29 : Analytical HPLC of the [DyL" ${ }^{4 c}$ complex. Column: Vision HT C18 HL $5 \mu 250 \times 4.6 \mathrm{~mm}$. Gradient: 100\% $\mathrm{H}_{2} \mathrm{O} 0-5 \mathrm{~min}$, 0-90\% ACN 5-15 min, 90\% ACN 15-20 min, 100\% $\mathrm{H}_{2} \mathrm{O} 20-25 \mathrm{~min}$. Flow: $1 \mathrm{~mL} / \mathrm{min}$. retention time $=14.733 \mathrm{~min}$. 


\begin{tabular}{llll} 
Analysis Info & & Acquisition Date & $31 / 01 / 201810: 39: 48$ \\
Sample Name & NH153f1 & Instrument/Ser\# & maXis 255552.00086 \\
Analysis Name & X039735CYC.d & Method & Positif.m \\
\hline
\end{tabular}

\begin{tabular}{|c|c|c|c|c|c|}
\hline \multicolumn{6}{|c|}{ Acquisition Parameter } \\
\hline $\begin{array}{l}\text { Source Type } \\
\text { Scan Begin }\end{array}$ & $\begin{array}{l}\text { ESI } \\
50 \mathrm{~m} / \mathrm{z}\end{array}$ & $\begin{array}{l}\text { Ion Polarity } \\
\text { Set Capillary }\end{array}$ & $\begin{array}{l}\text { Positve } \\
4500 \mathrm{~V}\end{array}$ & Set Nabullzer & $\begin{array}{l}0.6 \mathrm{Bar} \\
200^{\circ} \mathrm{C}\end{array}$ \\
\hline Scan End & $2500 \mathrm{~m} / \mathrm{z}$ & Set Coillsion Cell RF & $1800.0 \mathrm{VPp}$ & Set Doy Gas & $7.0 \mathrm{mmir}$ \\
\hline
\end{tabular}

Page | 25
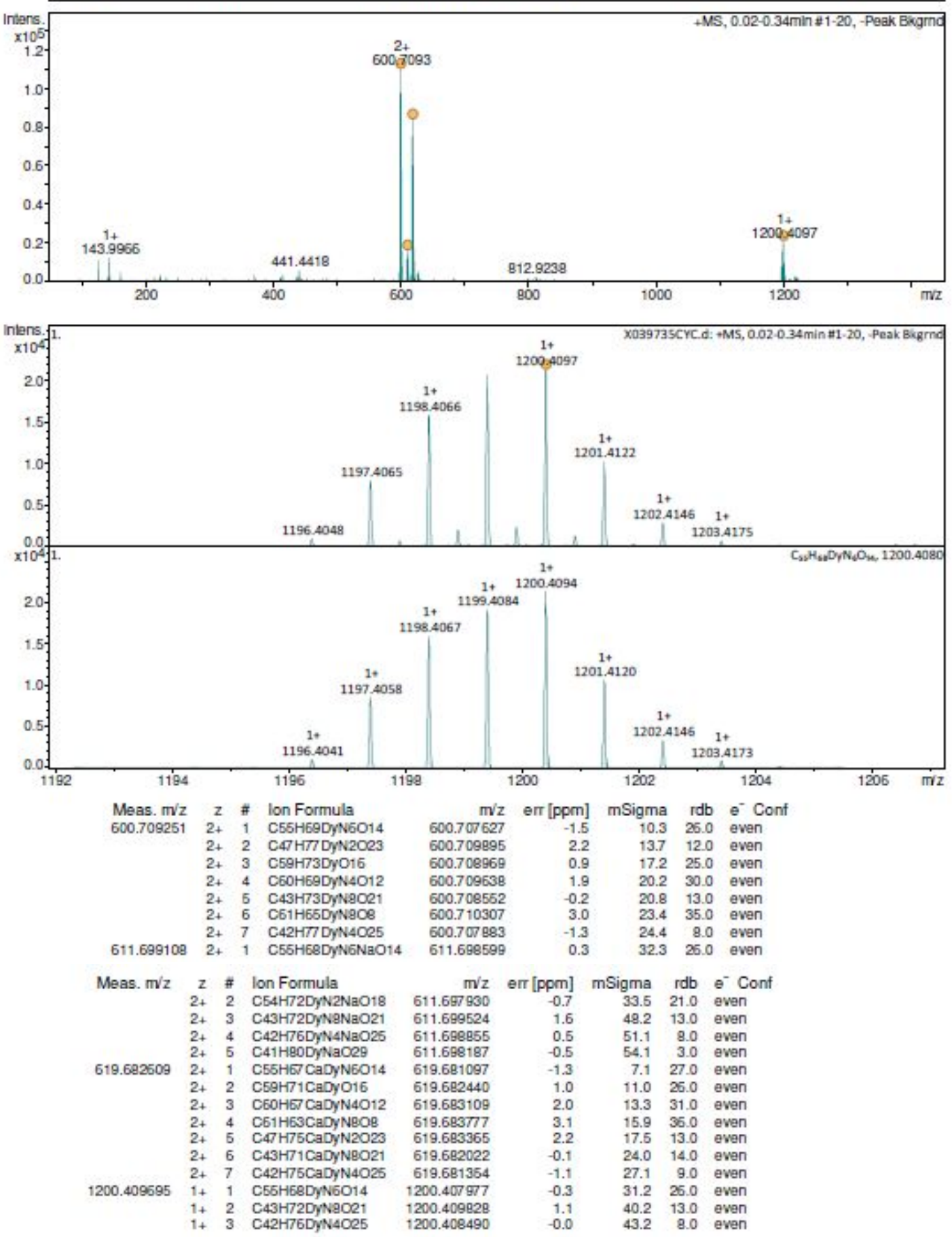

Figure S30 : HRMS of the $\left[D^{2} L^{4 c}\right]$ complex. 
Page | 26
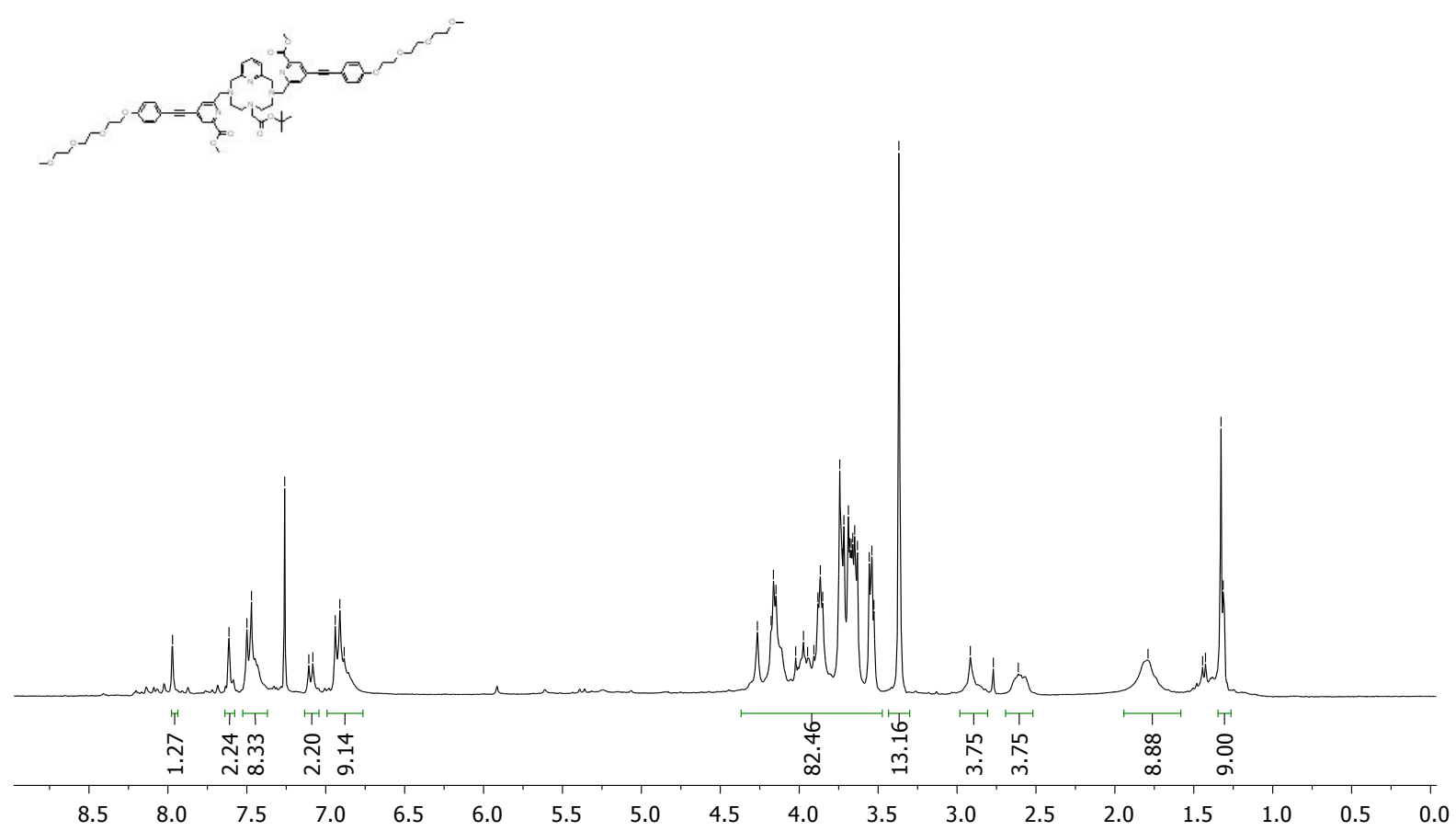

Figure S31 : ${ }^{1} \mathrm{H}$ NMR of compound 9.

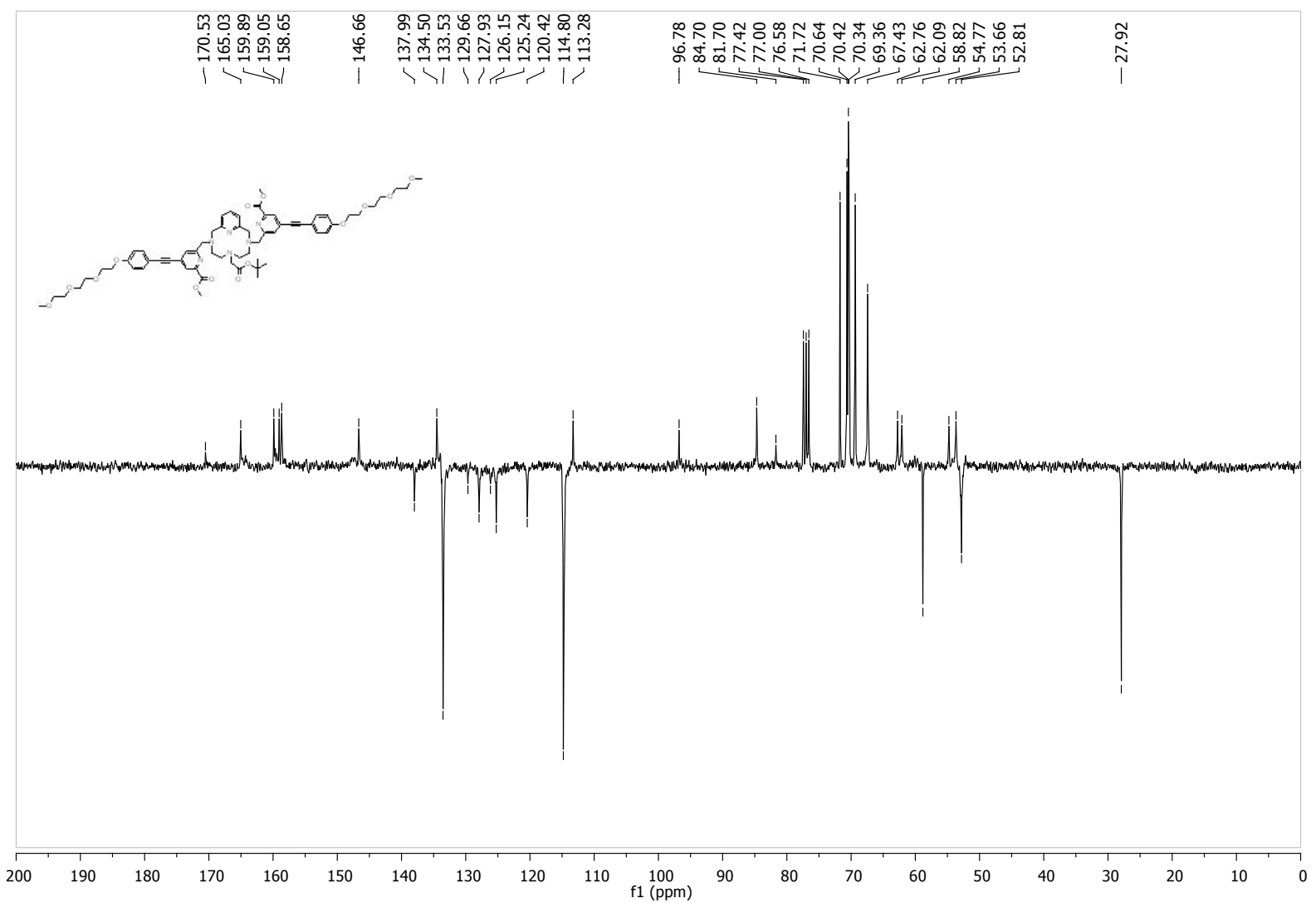

Figure S32: ${ }^{13} \mathrm{C}$ NMR of compound 9. 
Page | 27

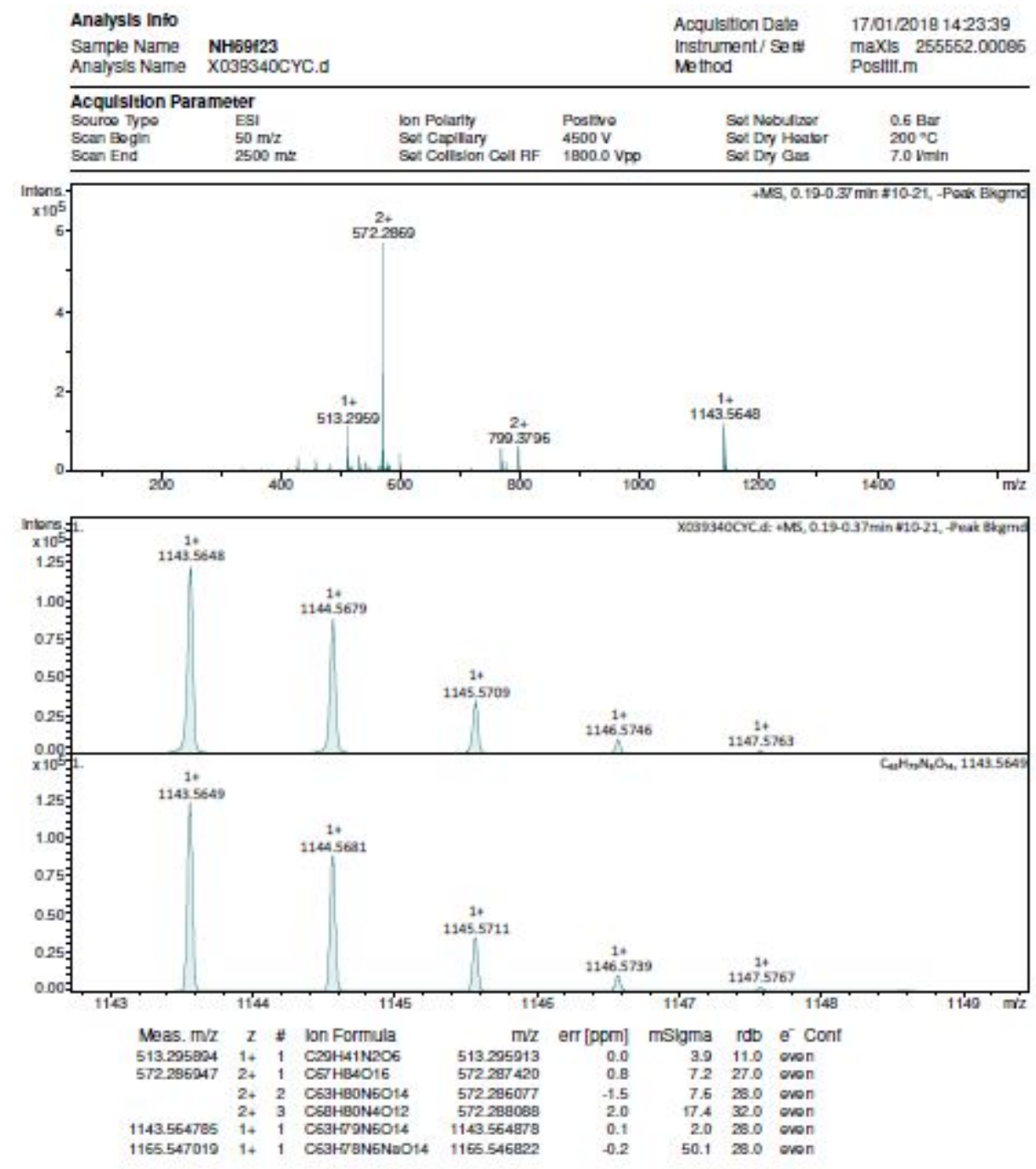

Figure S33 : HRMS of compound 9. 
Page | 28

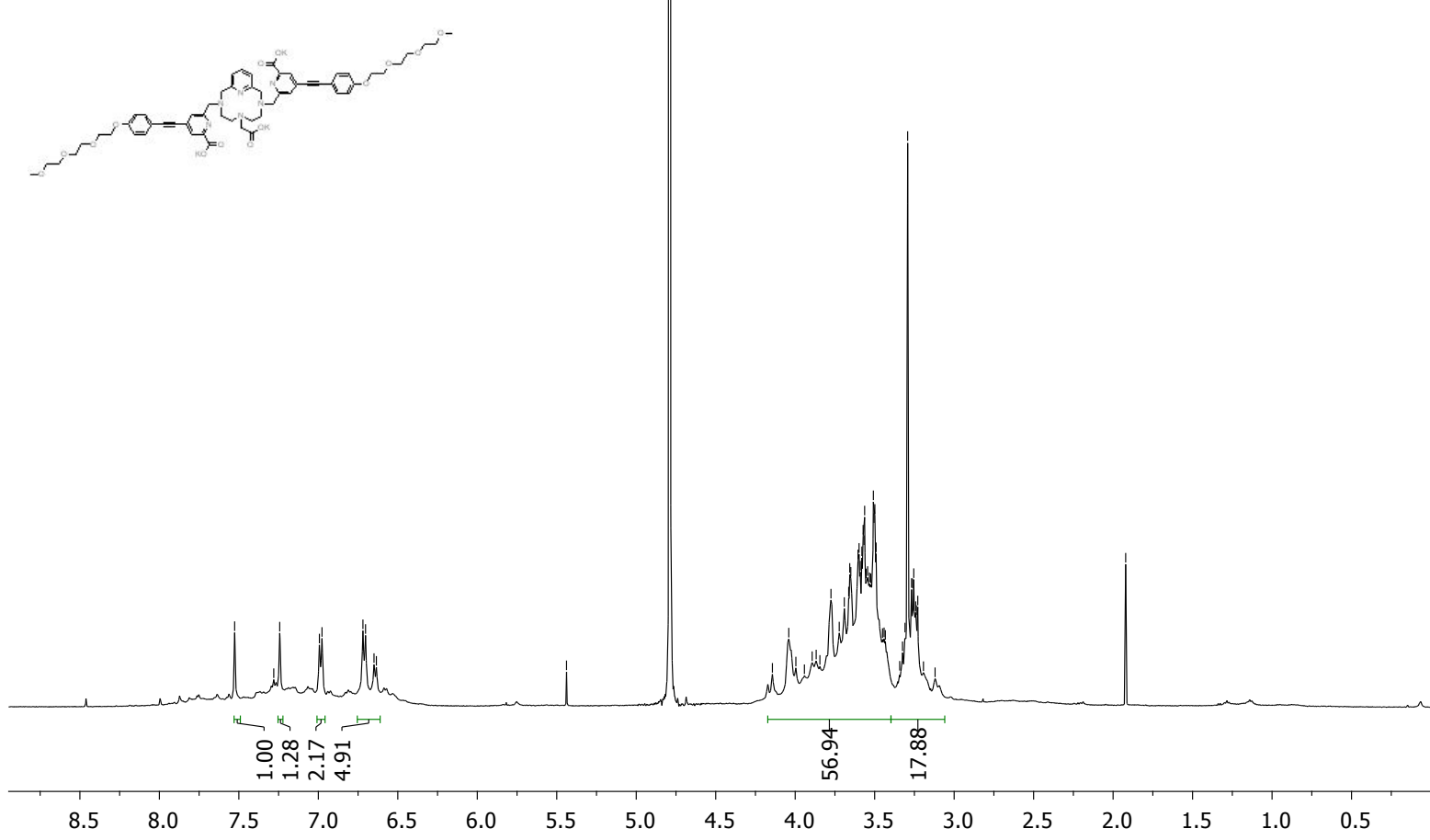

Figure S34 : ${ }^{1} \mathrm{H}$ NMR of compound $\mathrm{L}^{4 a}$.

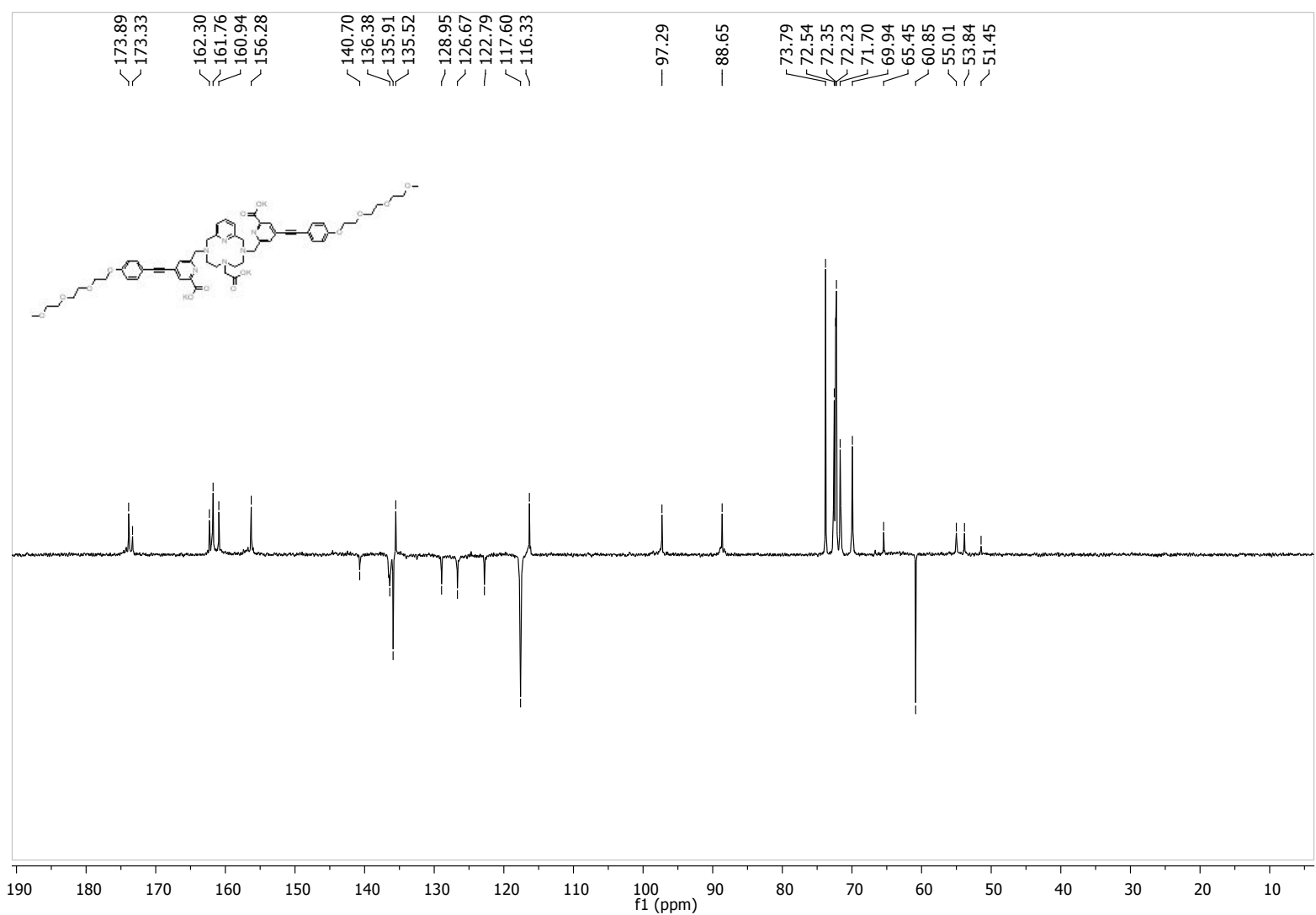

Figure $\mathbf{S 3 5}:{ }^{13} \mathrm{C}$ NMR of compound $\mathrm{L}^{4 a}$. 


\begin{tabular}{llll} 
Analysis Info & & Acquisition Date & $17 / 01 / 201817: 59: 27$ \\
Sample Name & NH144f2 & Instrument/Ser\# & maXis 255552.00086 \\
Analysis Name & X039341CYC_14592.d & Method & Positif.m \\
\hline
\end{tabular}

\begin{tabular}{llllll}
\hline Acquisition Parameter & & & & \\
Source Type & ESI & Ion Polarly & Positwe & Set Nebullzer & 0.6 Bar \\
Scan Begin & $50 \mathrm{~m} / \mathrm{z}$ & Set Capllary & $4500 \mathrm{~V}$ & Set Dry Healer & $200^{\circ} \mathrm{C}$ \\
Scan End & $2500 \mathrm{mz}$ & Set Collsion Cel RF & $1800.0 \mathrm{Vpp}$ & Set Dry Gas & 7.0 Umin
\end{tabular}

Page | 29
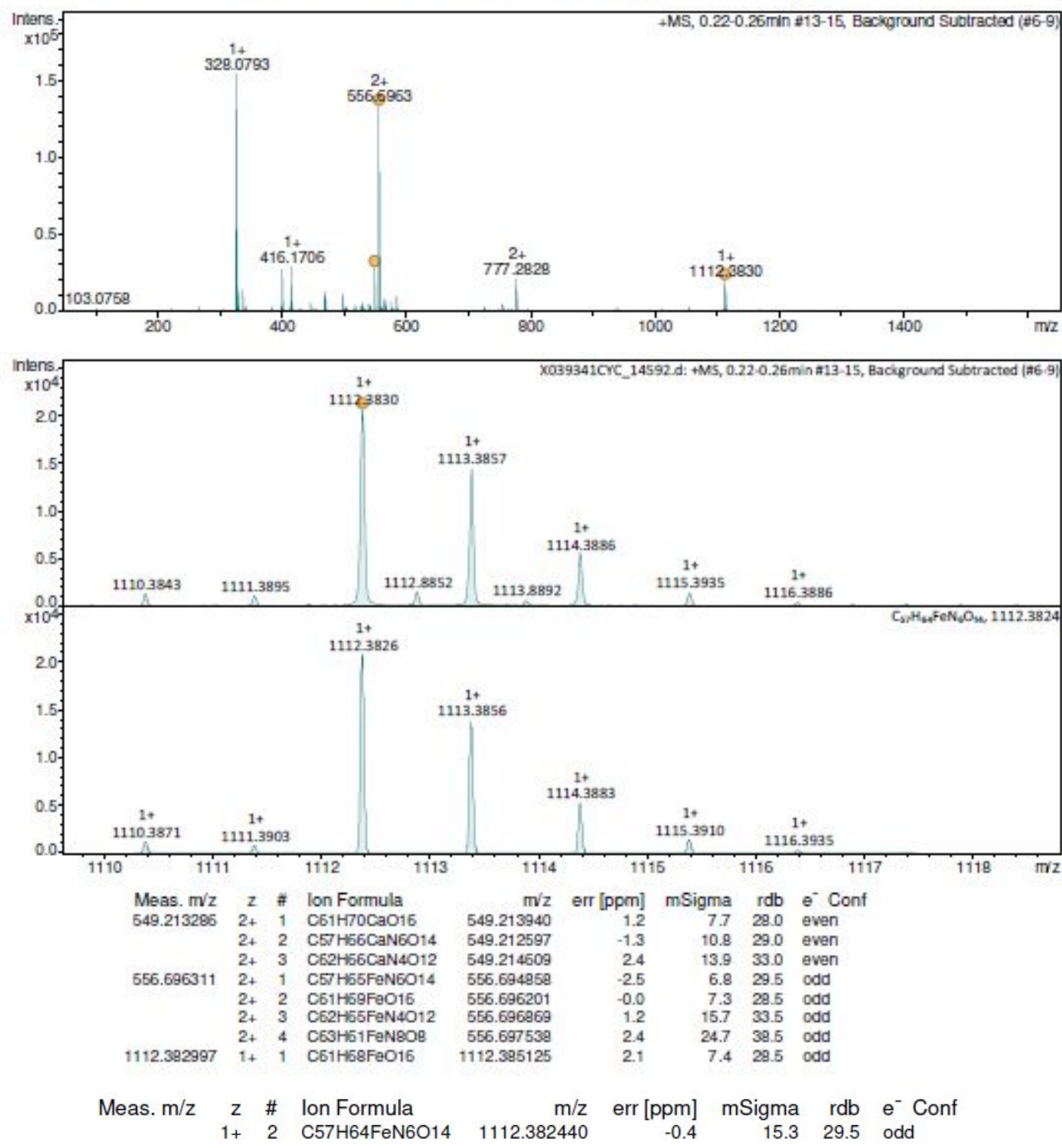

Figure S36 : HRMS of compound $L^{4 a}$. 
$\mathrm{mV}$

Page | 30

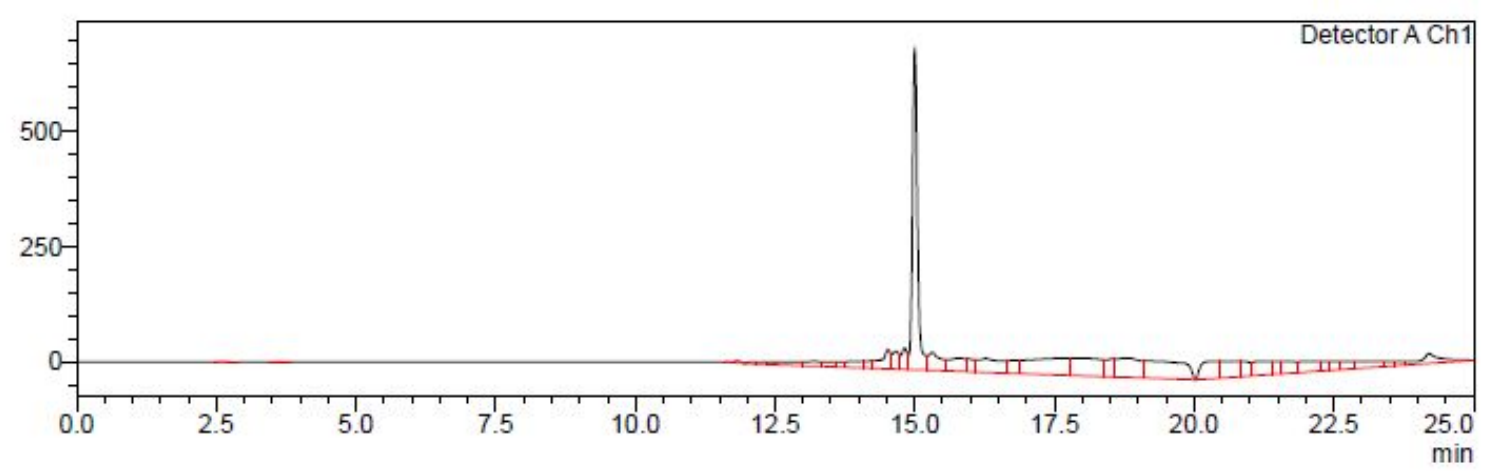

Figure S37 : Analytical HPLC of the [EuL ${ }^{4 a}$ ] complex. Column: Vision HT C18 HL $5 \mu 250 \times 4.6 \mathrm{~mm}$. Gradient: $100 \% \mathrm{H}_{2} \mathrm{O} 0-5 \mathrm{~min}$, $0-90 \%$ ACN 5-15 min, 90\% ACN 15-20 min, 100\% $\mathrm{H}_{2} \mathrm{O} 20-25 \mathrm{~min}$. Flow: $1 \mathrm{~mL} / \mathrm{min}$. retention time $=15.000 \mathrm{~min}$. 


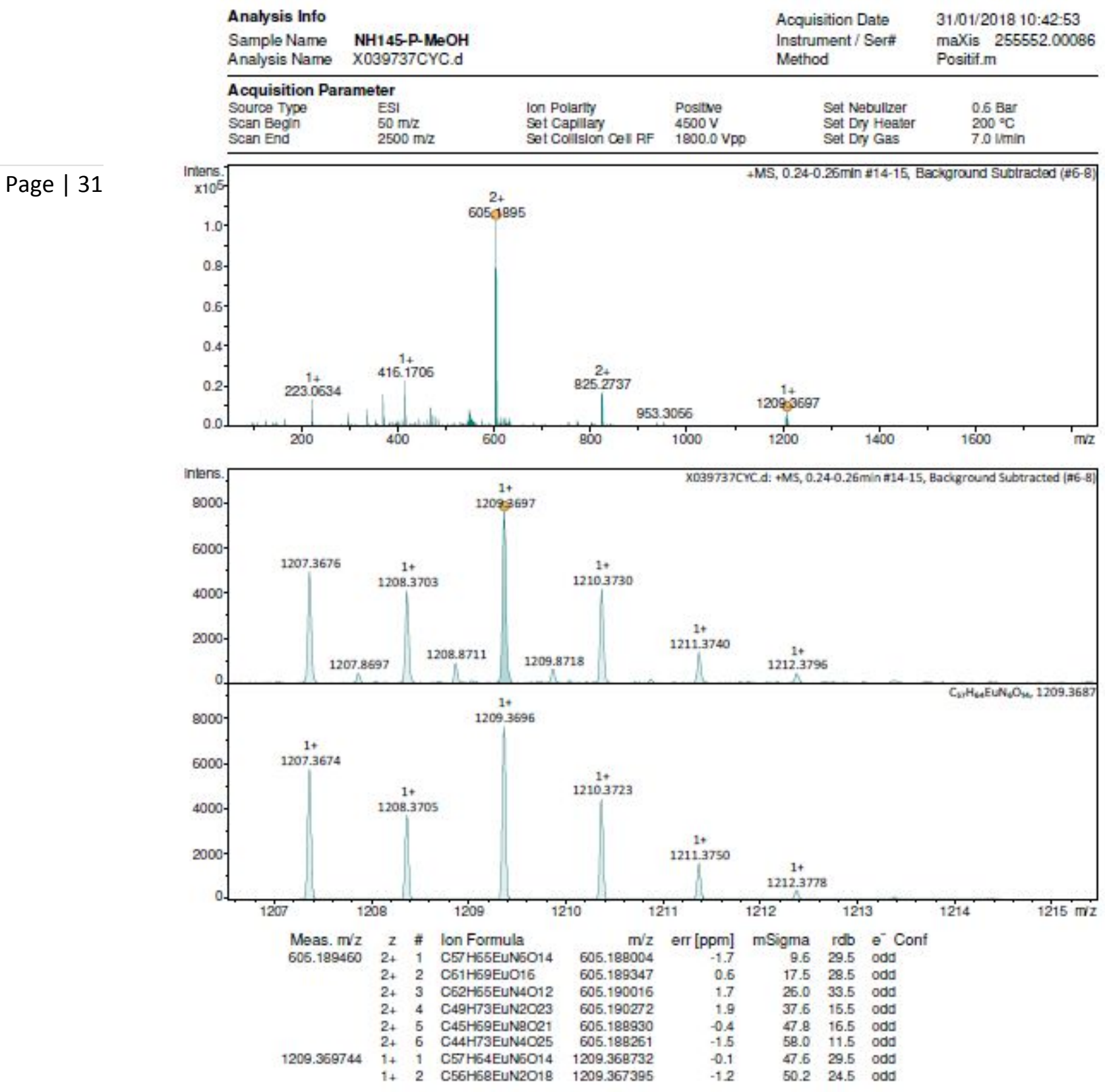

Figure S38 : HRMS of the [EuL $\left.{ }^{4 a^{\prime}}\right]$ complex. 


\section{Photophysical measurements.}

Page | 32

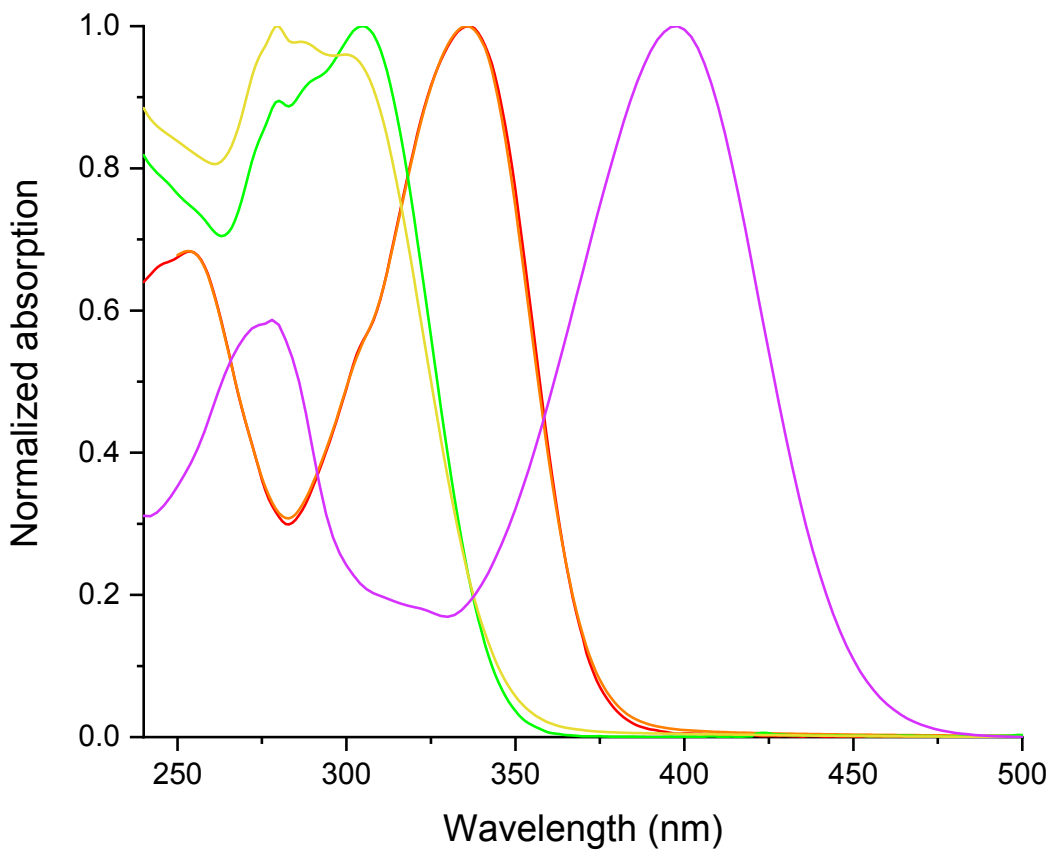

Figure S39: Normalized absorption spectra of $\left[\mathrm{EuL}^{4 \mathrm{a}}\right]$ (red), $\left[\mathrm{SmL}^{4 \mathrm{a}}\right]$ (orange), [YbL $\left.{ }^{4 b}\right]$ (purple), [TbL $\left.{ }^{4 c}\right]$ (green) and [DyL $\left.{ }^{4 c}\right]$ (yellow) in $\mathrm{MeOH}$ at room temperature.

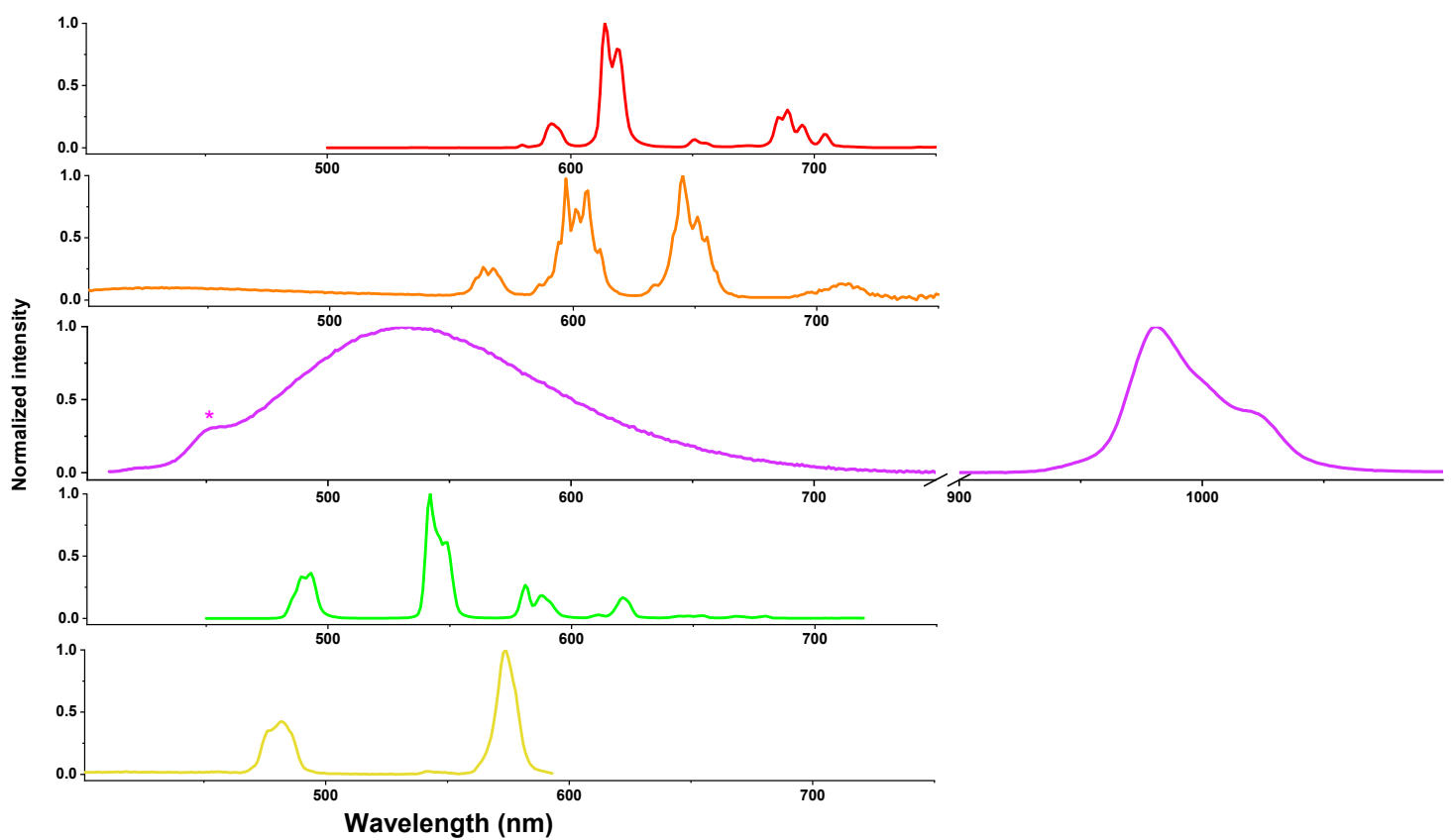

Figure $\mathbf{S 4 0}$ : Normalized emission spectra of [EuL $\left.{ }^{4 a}\right]\left(\right.$ red, $\lambda_{\mathrm{ex}}=336 \mathrm{~nm}$ ), [SmL ${ }^{4 a}$ ] (orange, $\left.\lambda_{\mathrm{ex}}=340 \mathrm{~nm}\right),\left[\mathrm{YbL}^{4 b}\right]\left(\mathrm{purple}^{\mathrm{e}}, \lambda^{\mathrm{ex}}=\right.$ $395 \mathrm{~nm}$ ), [TbL ${ }^{4 c}$ ] (green, $\lambda_{\mathrm{ex}}=305 \mathrm{~nm}$ ) and [DyL ${ }^{4 c}$ ] (yellow, $\lambda_{\mathrm{ex}}=310 \mathrm{~nm}$ ) in $\mathrm{MeOH}$ at room temperature. Note that the * indicates the Raman band of methanol $\left(v_{\mathrm{ex}}-v_{\mathrm{raman}}=3290 \mathrm{~cm}^{-1}\right)$. 
Page | 33

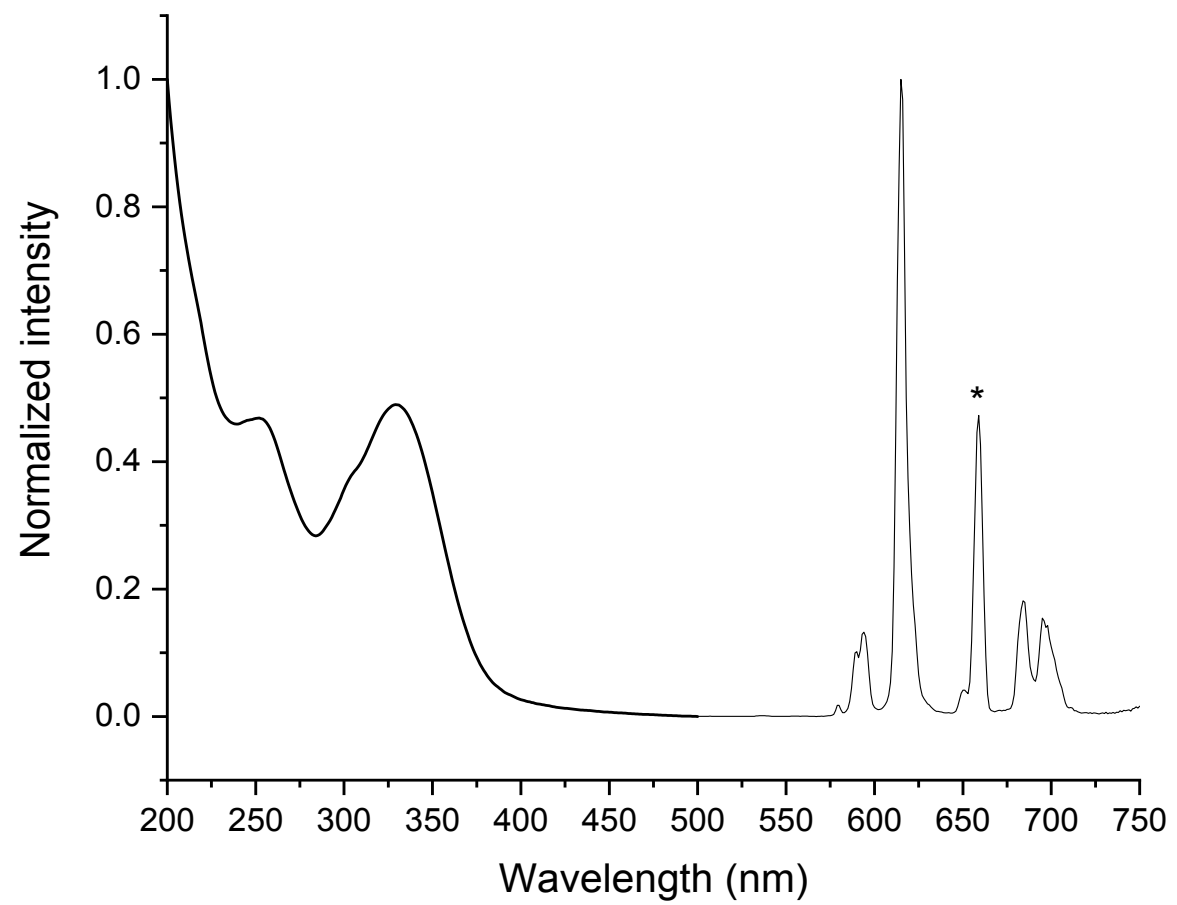

Figure S41: Normalized absorption (bold) and emission (thin, $\lambda_{\mathrm{ex}}=329 \mathrm{~nm}$ ) spectra in visible of [EuL 4a'] (in diluted water solution, at room temperature). The * represents the second harmonic scattering.

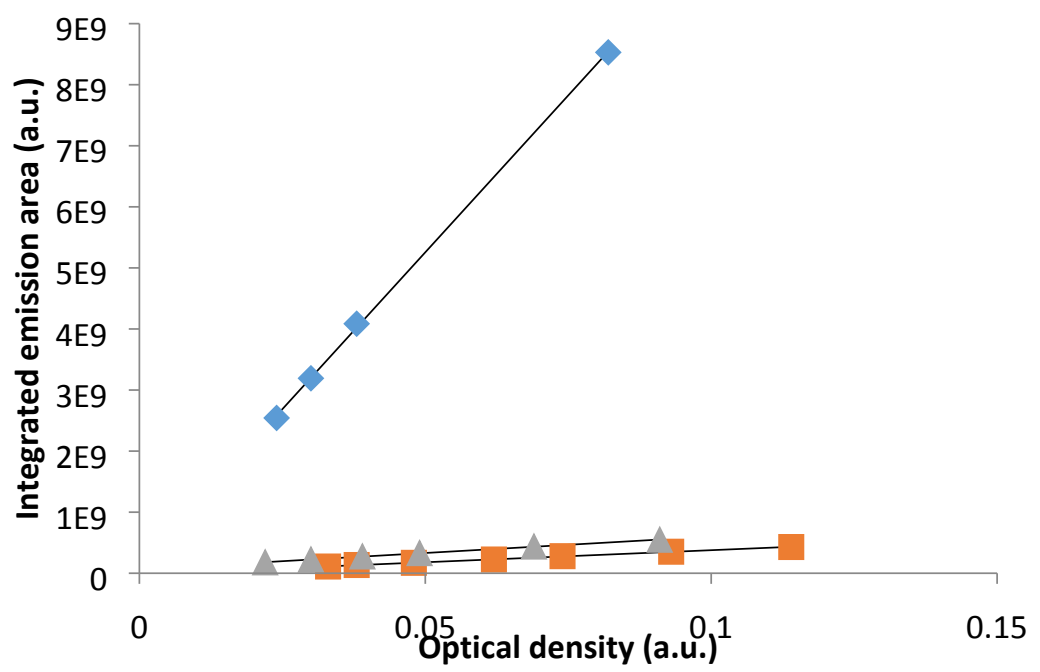

Figure S42: Determination of the quantum yield of [DyL $\left.{ }^{4 c}\right]$ in $\mathrm{MeOH}$ (grey), $\mathrm{H}_{2} \mathrm{O}$ (orange), and the reference quinine sulfate in a $1 \mathrm{~N}$ aqueous solution of sulfuric acid (blue). The straight lines correspond to the linear fit. 
Page | 34

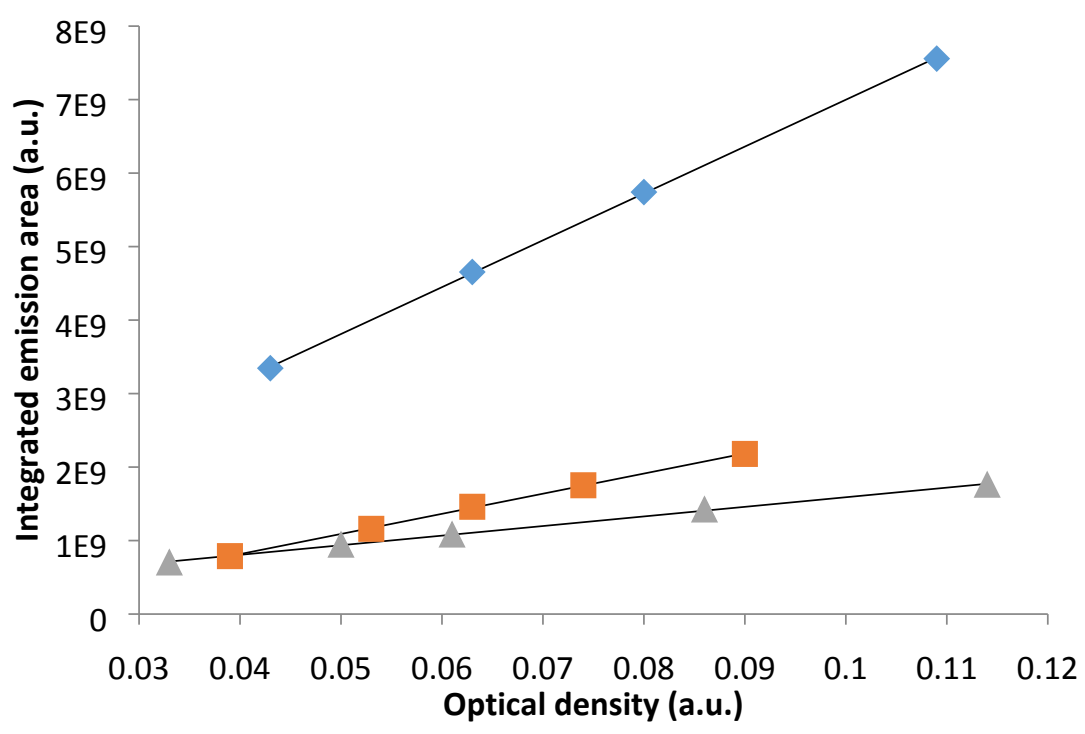

Figure S43: Determination of the quantum yield of [EuL $\left.\mathrm{La}^{\prime}\right]$ in $\mathrm{MeOH}$ (grey), $\mathrm{H}_{2} \mathrm{O}$ (orange), and the reference quinine sulfate in a $1 \mathrm{~N}$ aqueous solution of sulfuric acid (blue). The straight lines correspond to the linear fit.

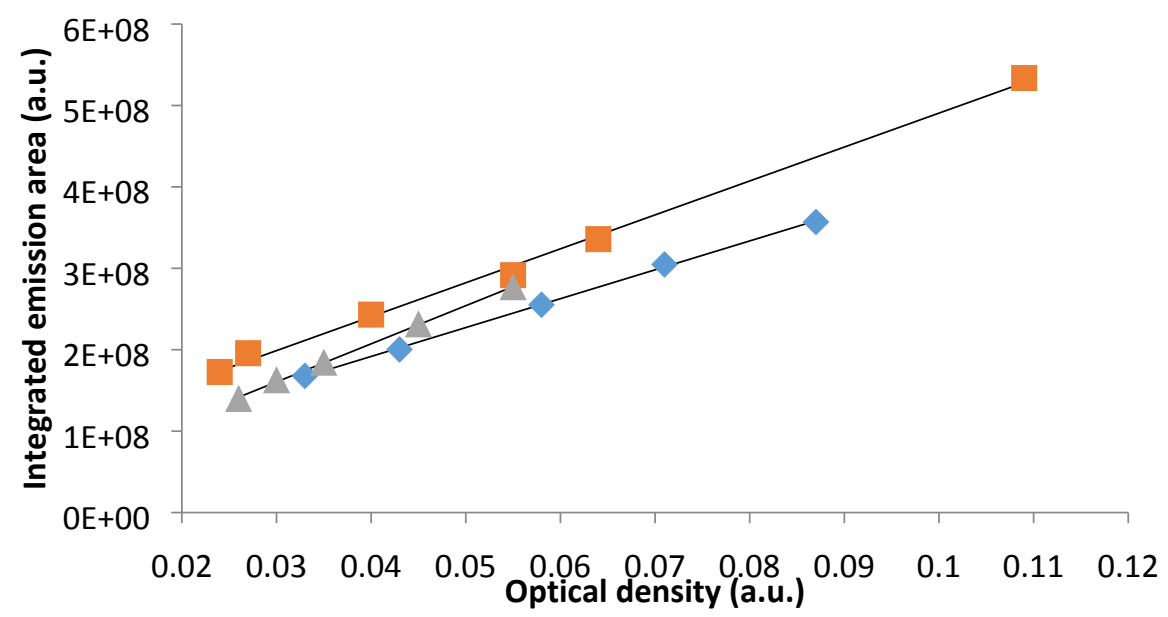

Figure S44: Determination of the quantum yield of $\left[\mathrm{TbL}^{4 c}\right]$ in $\mathrm{MeOH}$ (grey), $\mathrm{H}_{2} \mathrm{O}$ (orange), and the reference quinine sulfate in a $1 \mathrm{~N}$ aqueous solution of sulfuric acid (blue). The straight lines correspond to the linear fit. 
Page | 35

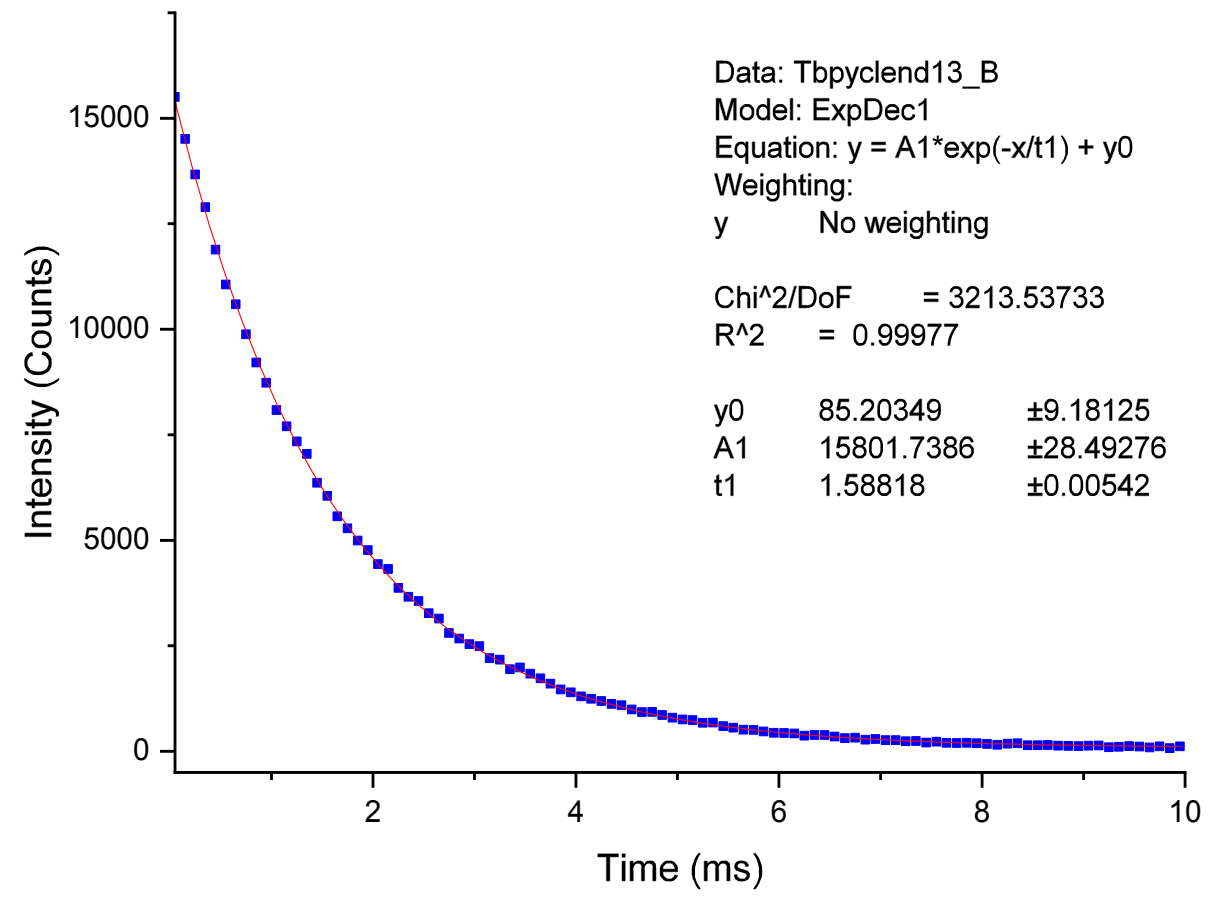

Figure S45: Lifetime measurement of $\left[\mathrm{TbL}^{4 c}\right]$ in $\mathrm{MeOH}$ (blue) and the fitting curve (red).

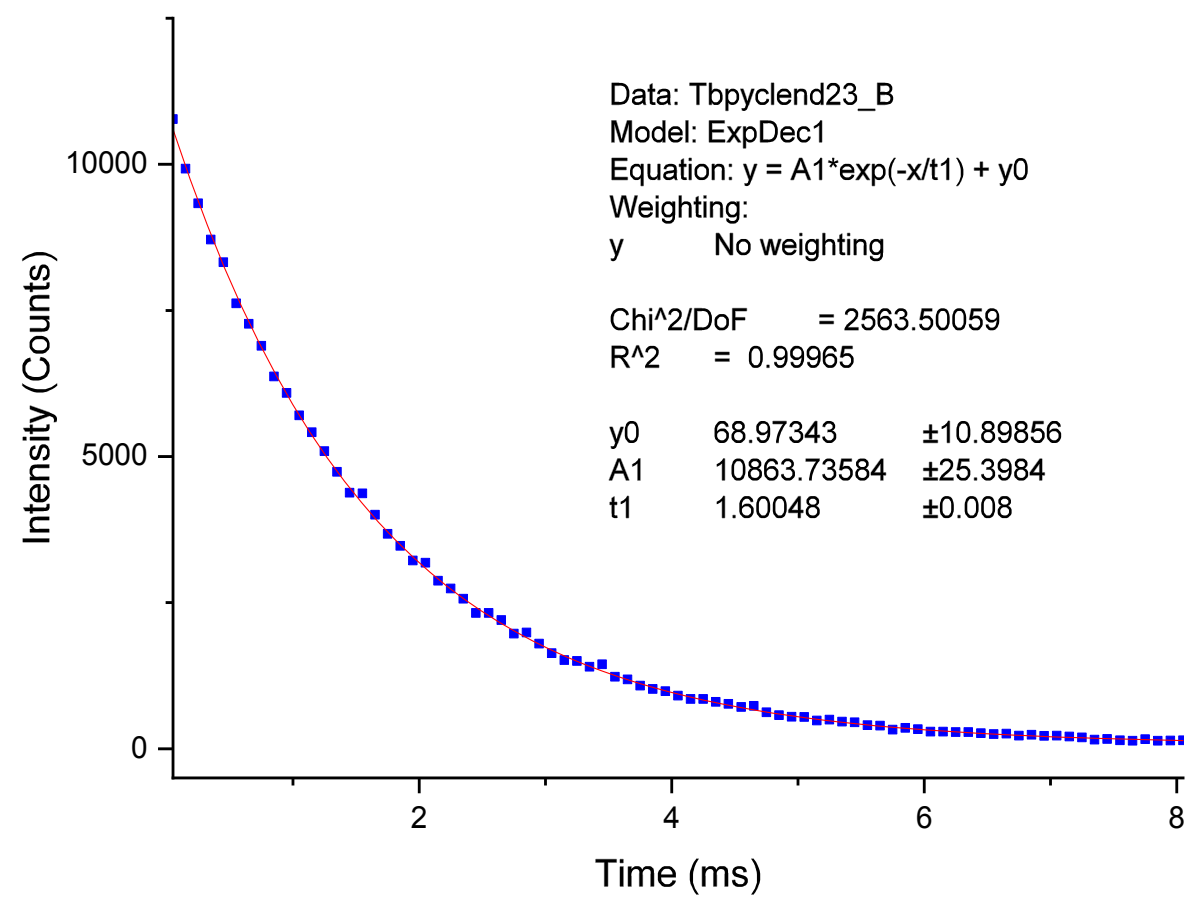

Figure S46: Lifetime measurement of [ $\left.\mathrm{TbL}^{4 \mathrm{c}}\right]$ in $\mathrm{H}_{2} \mathrm{O}$ (blue) and the fitting curve (red). 
Page | 36

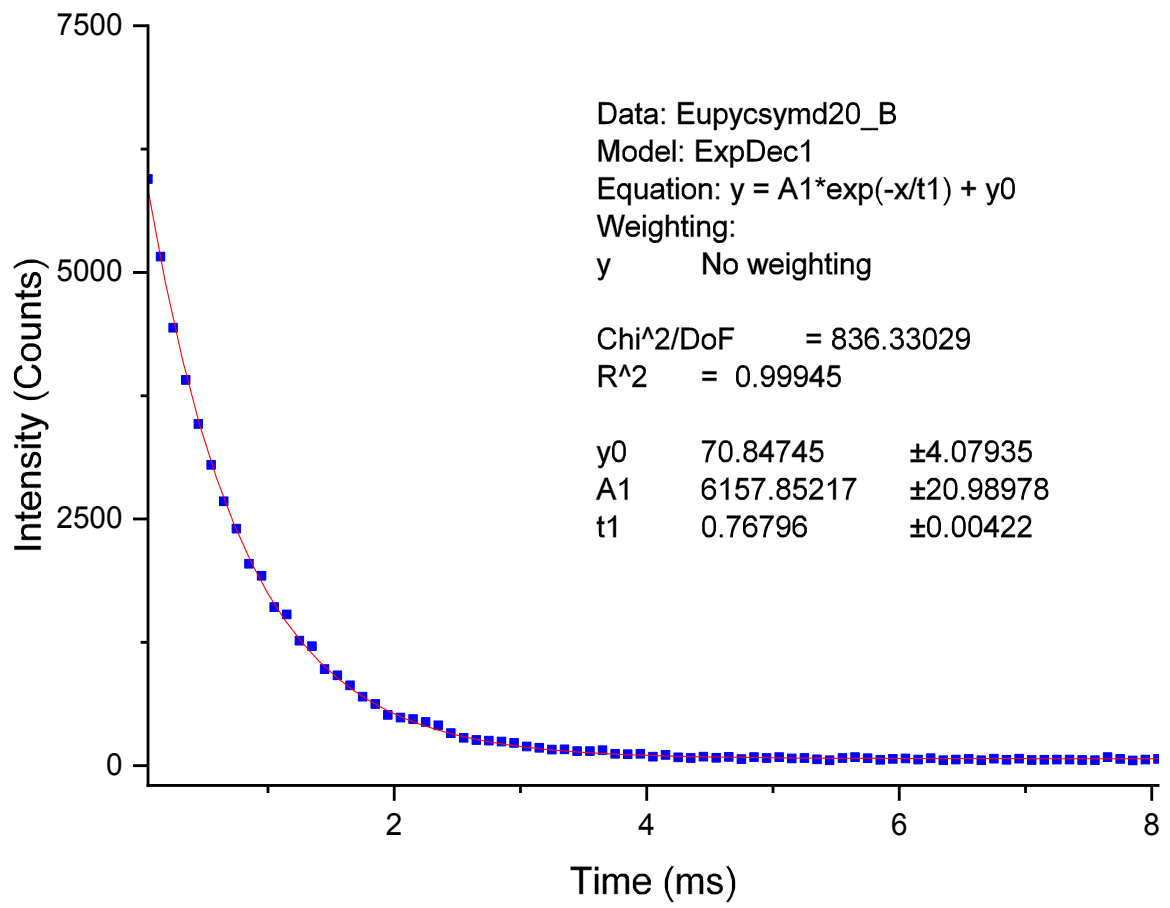

Figure S47: Lifetime measurement of $\left[\mathrm{EuL}^{4 \mathrm{a}^{\prime}}\right]$ in $\mathrm{H}_{2} \mathrm{O}$ (blue) and the fitting curve (red).

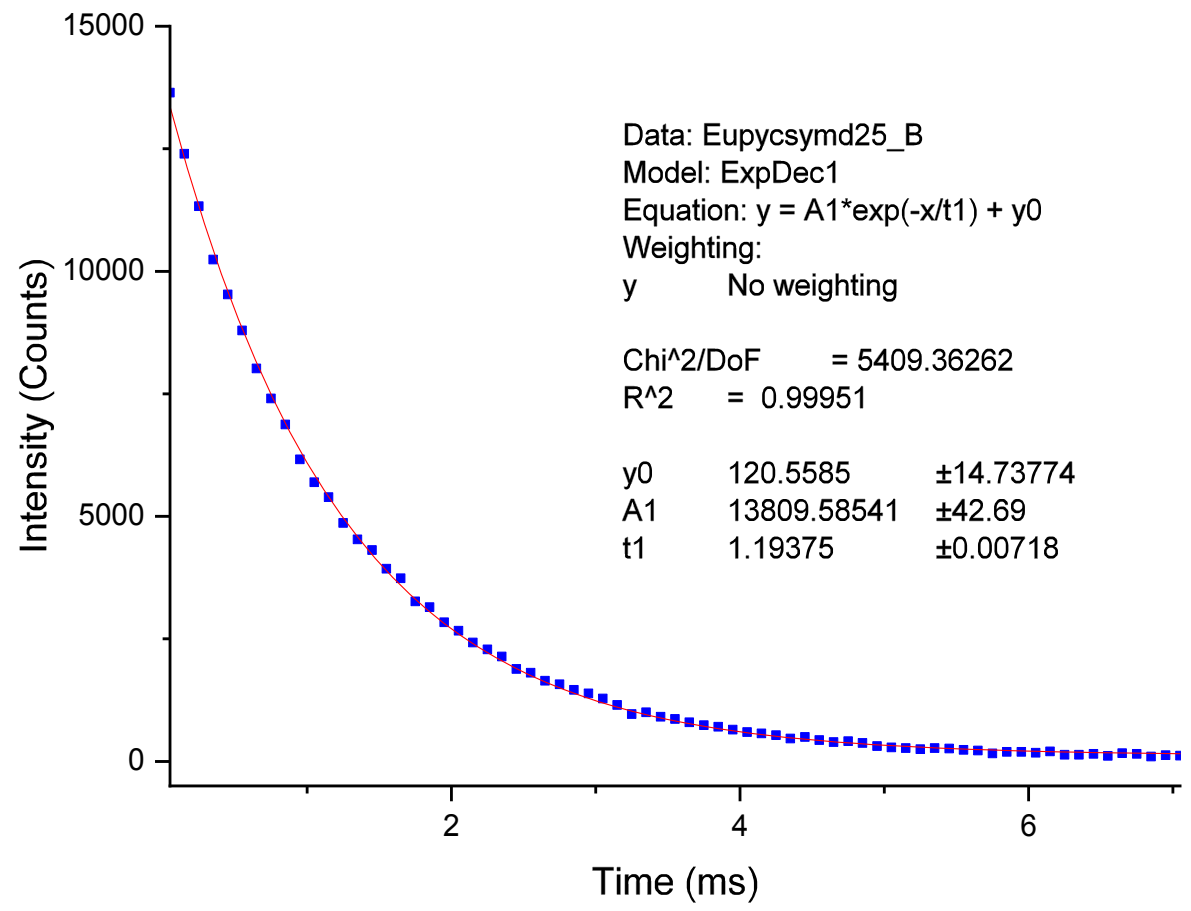

Figure S48: Lifetime measurement of [EuL $\left.{ }^{4 a}\right]$ in $\mathrm{MeOH}$ (blue) and the fitting curve (red). 
Page | 37

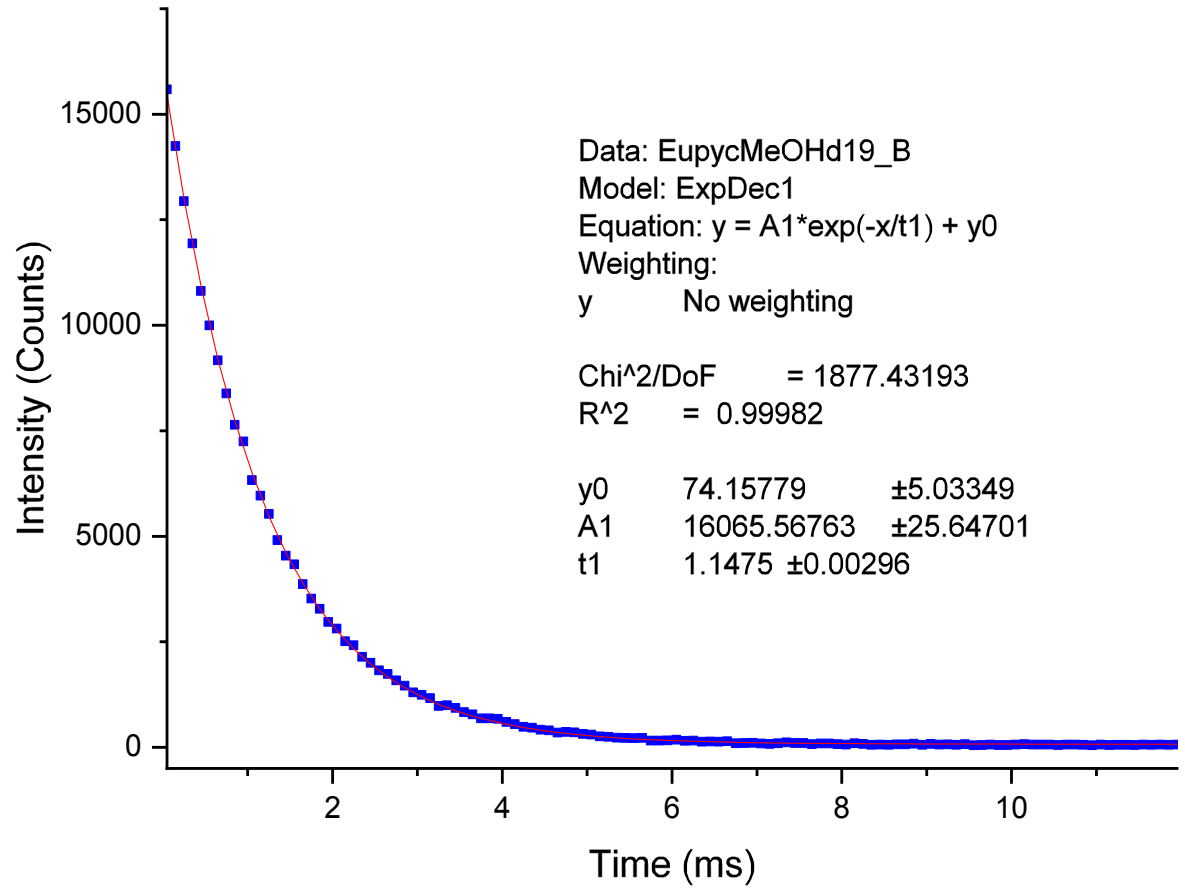

Figure S49: Lifetime measurement of $\left[\mathrm{EuL}^{4 \mathrm{a}}\right]$ in $\mathrm{MeOH}$ (blue) and the fitting curve (red).

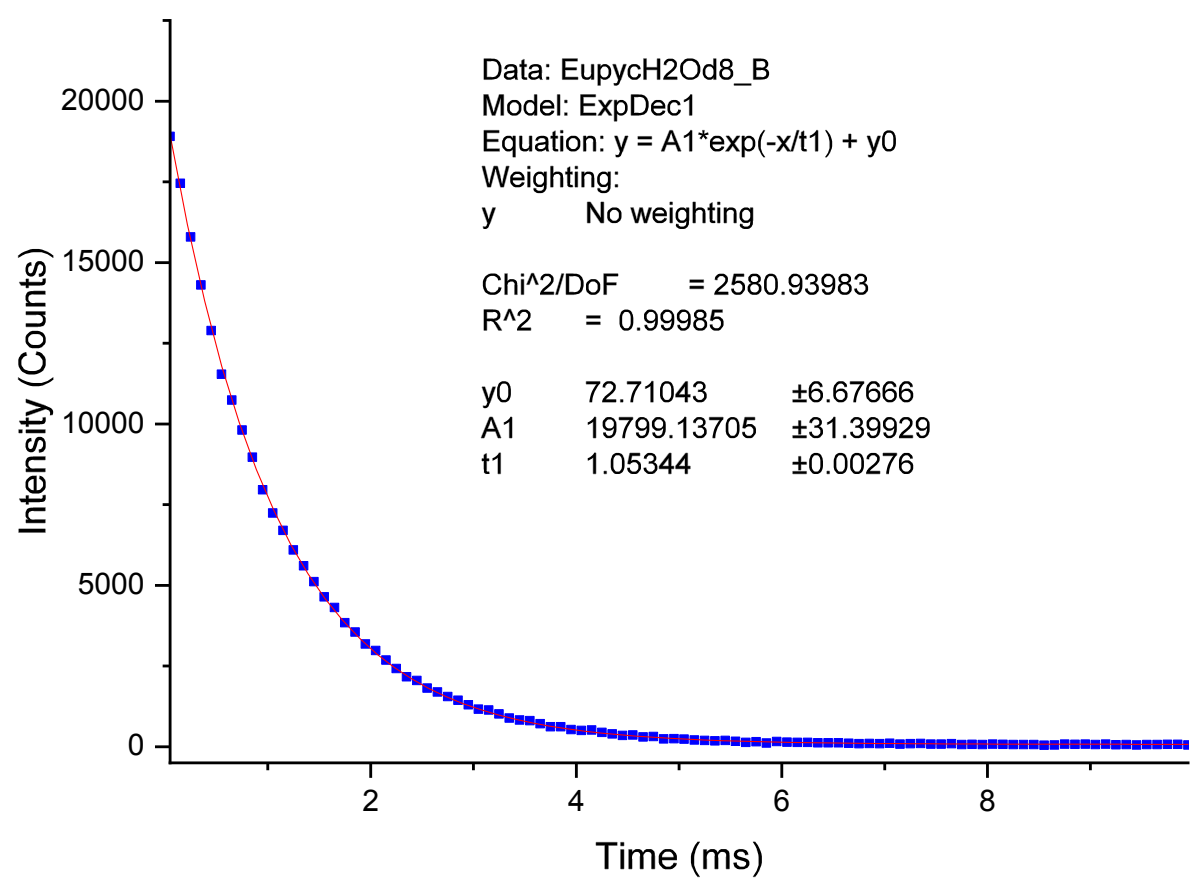

Figure S50: Lifetime measurement of [EuL $\left.{ }^{4 a}\right]$ in $\mathrm{H}_{2} \mathrm{O}$ (blue) and the fitting curve (red). 
Page | 38

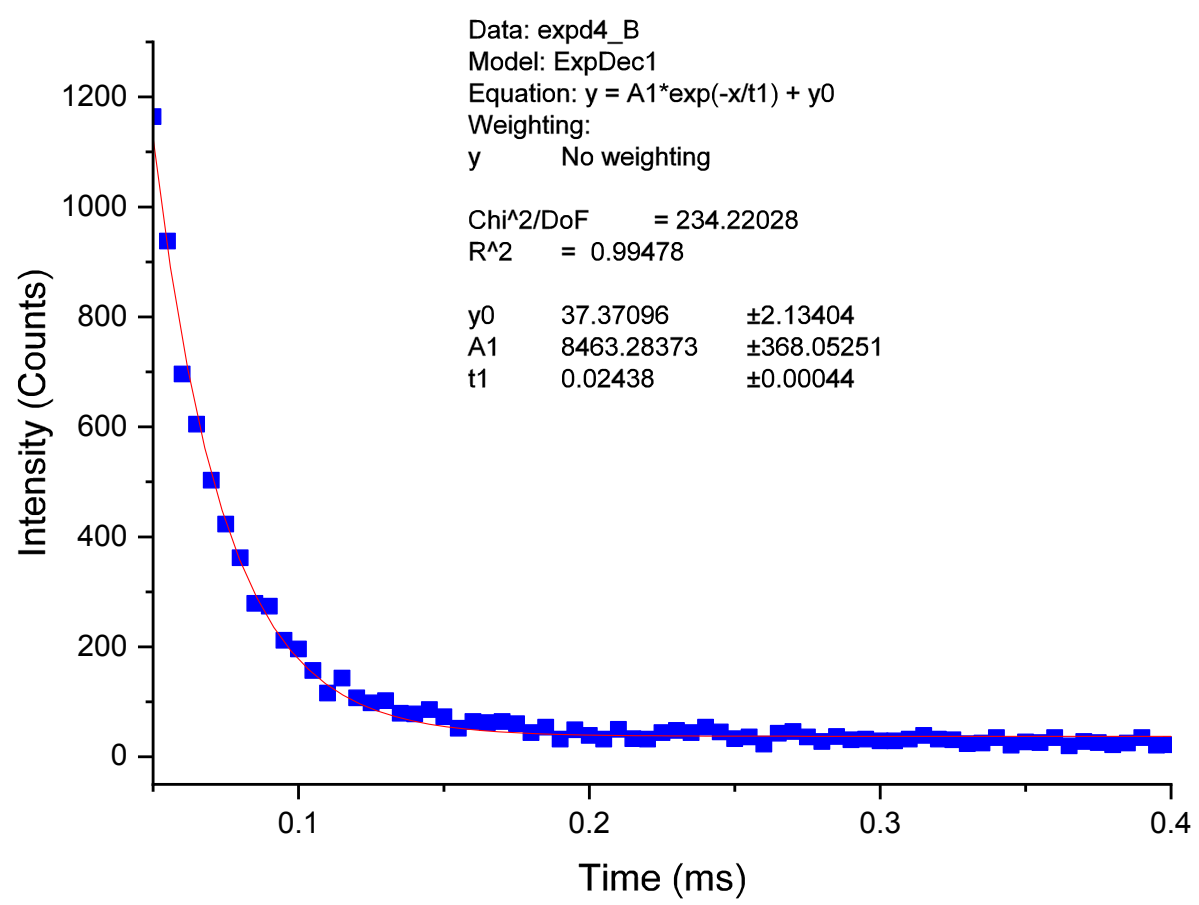

Figure S51: Lifetime measurement of $\left[\mathrm{SmL}^{4 \mathbf{b}}\right]$ in $\mathrm{MeOH}$ (blue) and the fitting curve (red).

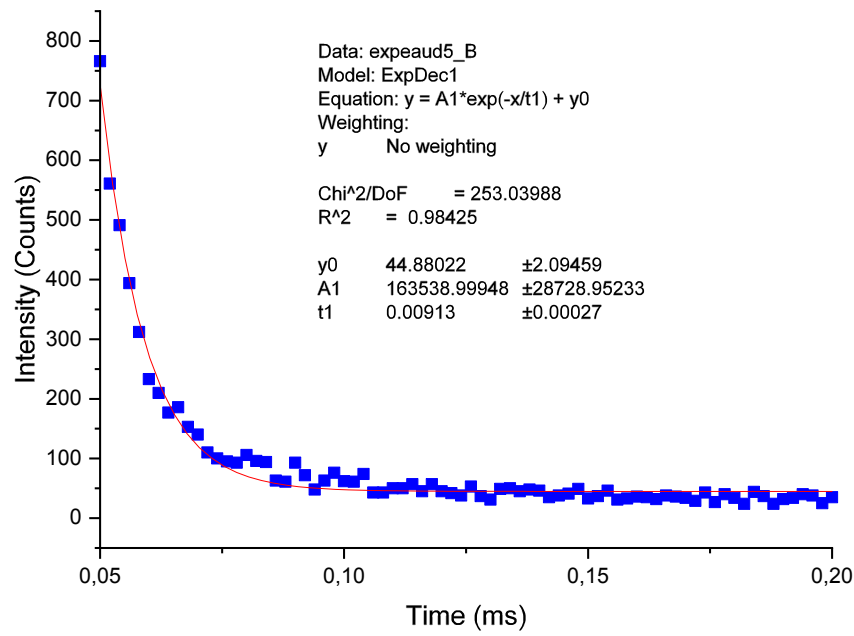

Figure S52: Lifetime measurement of $\left[\mathrm{SmL}^{4 b}\right]$ in $\mathrm{H}_{2} \mathrm{O}$ (blue) and the fitting curve (red). 
Page | 39

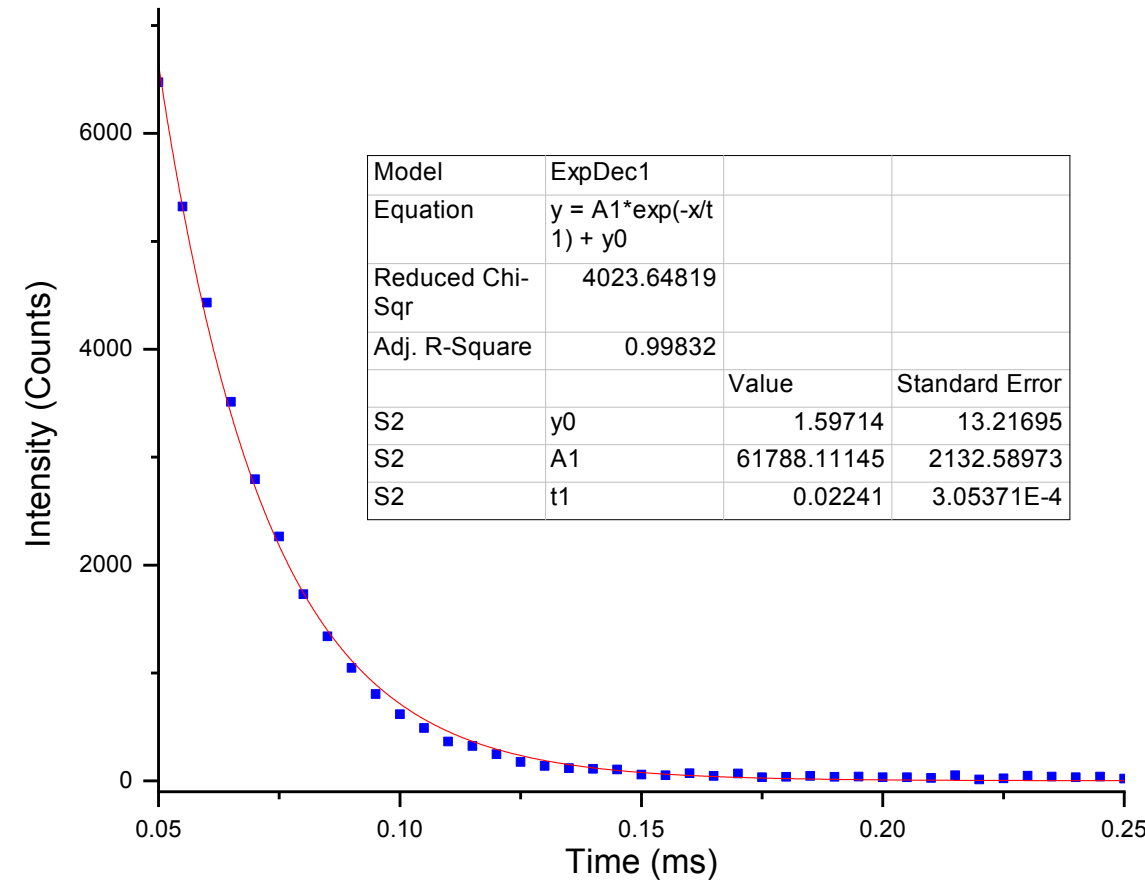

Figure S53: Lifetime measurement of $\left[\mathrm{Dy}^{4 c}\right]$ in $\mathrm{H}_{2} \mathrm{O}$ (blue) and the fitting curve (red). 